\title{
The sub-Cauchy Stokes Problem: Solvability Issues and Lagrange Multiplier Methods with Artificial Boundary Conditions
}

\author{
E. AHMED ${ }^{\mathrm{a}, \mathrm{b}, *}$, A. Ben $\mathrm{ABDA}^{\mathrm{c}}$ \\ ${ }^{a}$ Inria Paris, 2 rue Simone Iff, 75589 Paris, France \& Université Paris-Est, CERMICS (ENPC), 77455 Marne-la-Vallée 2, \\ France \\ ${ }^{b}$ Université Paris 13, UMR 7539, LAGA, 93430 Villetaneuse, France \\ ${ }^{c}$ Université Tunis El Manar, LR99ES20, LAMSIN-ENIT, B.P. 37, 1002 Tunis-Belvédère, Tunisia
}

\begin{abstract}
In contrast to the conventional Cauchy Stokes problems, in which the velocity and the stress force data are given on the accessible boundary, in the present paper, we reduce the accessible boundary data information and we consider a problem which deals only with shear stress data. We refer to this problem as a sub-Cauchy Stokes problem. This problem is ill-posed because of severe instability and even uniqueness is unknown. We first address the uniqueness issues associated with this problem. Resorting to the domain decomposition techniques together with the duplication process of Vogelius [1], we propose new Lagrange multiplier methods to solve the sub-Cauchy Stokes problem. These methods consist in recasting the problem in terms of interfacial equations, by equalizing two solutions of the sub-Cauchy Stokes problem using matching conditions defined on the inaccessible boundary. The matching is based on second order conditions and depends on the equations used to match the values of the unknowns on the inaccessible boundary. The underlying interfacial problems are then solved by iterative procedures in which coefficients can be optimized to improve convergence rates. A complete analysis of the methods is presented, and various numerical results illustrate the effectiveness and the performance of the proposed approaches.
\end{abstract}

Keywords: Inverse problem, Sub-Cauchy Stokes system, Uniqueness, Ventcell boundary conditions, Alternating method, Noise, Convergence factor.

\section{Introduction}

This work is concerned with the data completion problem associated with the Stokes system. This is a strongly ill-posed inverse problem. To describe the inverse problem, we let $\Omega$ be a bounded and simply connected domain of $\mathbb{R}^{2}$, with Lipschitz boundary $\Gamma=\partial \Omega$ made up of three connected disjoint open subsets satisfying $\cup_{i=\mathrm{I}, \mathrm{D}, \mathrm{C}} \overline{\Gamma_{i}}=\Gamma$. The portion $\Gamma_{\mathrm{C}}$ is considered as accessible to measures while $\Gamma_{\mathrm{I}}$ is the unreachable boundary and no data are thus available on it. Let us denote as usual, by $\mathbf{n}$ the outward unit normal vector and by $\boldsymbol{\tau}$ the unit tangent vector to the boundary $\Gamma$. For any vector field $\mathbf{v}$ on $\partial \Omega$, we shall denote by $\mathbf{v}_{\boldsymbol{\tau}}$ the projection of $\mathbf{v}$ on the tangent hyperplane to $\Gamma$. In other words $\mathbf{v}_{\boldsymbol{\tau}}=\mathbf{v}-(\mathbf{v} \cdot \mathbf{n}) \mathbf{n}$.

The data completion problem we consider consists in finding the solution $(\mathbf{u}, p)$ of the incompressible Stokes equations

$$
\begin{aligned}
\nabla \cdot \mathbf{u}=0 & \text { in } \Omega, \\
-\Delta \mathbf{u}+\nabla p=0 & \text { in } \Omega,
\end{aligned}
$$

\footnotetext{
* Corresponding author

Email addresses: elyes.ahmed@inria.fr (E. AHMED), amel.benabda@enit.rnu.tn (A. Ben ABDA)
} 
and subject to the following physical boundary conditions

$$
\begin{aligned}
& \boldsymbol{\tau} \cdot 2 \mathbf{D}(\mathbf{u}) \mathbf{n}=\varphi \quad \text { on } \Gamma_{\mathrm{C}}, \\
& \mathbf{u}=\Phi \quad \text { on } \Gamma_{\mathrm{C}}, \\
& \mathbf{u}=0 \quad \text { on } \Gamma_{\mathrm{D}} \text {. }
\end{aligned}
$$

Here, $\Phi$ is a shearing flow, i.e., a flow wherein $\mathbf{u}=\mathbf{u}_{\boldsymbol{\tau}}$, and $\varphi$ is the shear stress component. The deformation rate tensor $\mathbf{D}(\mathbf{u})$ is given by $\mathbf{D}(\mathbf{u}) \stackrel{\text { def }}{=}\left(\nabla \mathbf{u}+\nabla^{T} \mathbf{u}\right) / 2$ (i.e., the symmetric part of the gradient). We denote by $\boldsymbol{\sigma}(\mathbf{u}, p)$ the stress tensor, i.e., $\boldsymbol{\sigma}(\mathbf{u}, p) \stackrel{\text { def }}{=} 2 \mathbf{D}(\mathbf{u})-p \mathbf{I}$, where $\mathbf{I}$ denotes the identity matrix.

The mathematical formulation corresponding to the data completion problem (1.1)-(1.5) is the wellknown Cauchy problem if the available data on the accessible boundary $\Gamma_{\mathrm{C}}$ refer to Cauchy data $(\mathbf{u}, \boldsymbol{\sigma}(\mathbf{u}, p) \mathbf{n})$, where the physical sense of $\boldsymbol{\sigma}(\mathbf{u}, p) \mathbf{n}$ is the Cauchy force acting on $\partial \Omega$. The components of the Cauchy force in the normal and tangential directions can be expressed by

$$
\begin{aligned}
& \mathbf{n} \cdot \boldsymbol{\sigma}(\mathbf{u}, p) \mathbf{n}=-p+\mathbf{n} \cdot 2 \mathbf{D}(\mathbf{u}) \mathbf{n} \text { (normal stress), } \\
& \boldsymbol{\tau} \cdot \boldsymbol{\sigma}(\mathbf{u}, p) \mathbf{n}=\boldsymbol{\tau} \cdot 2 \mathbf{D}(\mathbf{u}) \mathbf{n} \text { (shear stress). }
\end{aligned}
$$

This means that for classical Cauchy Stokes problem we should have the measurements of the quintuplet $\left.(\mathbf{u}, p, \mathbf{n} \cdot 2 \mathbf{D}(\mathbf{u}) \mathbf{n}, \boldsymbol{\tau} \cdot 2 \mathbf{D}(\mathbf{u}) \mathbf{n})\right|_{\Gamma_{\mathrm{C}}}$. However, regarding the available data given by the boundary conditions (1.3)-(1.4), one can easily show that the situation that we face here is not completely classical. In fact, the information of the pressure $p$ and the viscous stress $\mathbf{n} \cdot \mathbf{D}(\mathbf{u}) \mathbf{n}$ on $\Gamma_{\mathrm{C}}$ which are coupled in the normal stress (1.6) are unavailable.

The boundary conditions (1.3)-(1.4) are encountered in various industrial applications involving internal surfaces and interfaces to simulate flows near rough walls, such as in aerodynamics, in weather forecasts and in hæmodynamics, as well as perforated walls. In such surfaces collecting accurate measurements is difficult to do experimentally, and if it is possible, some measurements may be missing (see $[2,3,4,5]$ ). Mathematically, these measurements correspond to the so-called Navier friction, Navier slip, or simply known as Navier boundary conditions. Clearly, these measurements do not fit Fabre and Lebeau's Theorem that guarantees the uniqueness of the corresponding pair $(\mathbf{u}, p)$ (see [6]). In the literature, classical Cauchy problems have been extensively studied in recent years, and in particular the Stokes-Cauchy problem. Both solvability and stability have been investigated intensively $[6,7,8,9,10,11]$.

In terms of numerical methods, there have been various techniques applied to the Cauchy-Stokes equations. In [12], the authors propose a control-type method based on the least squares minimization problem with Cauchy-Stokes equations as constraints. Nowadays, a more appealing idea is given by the use of the energy-like formulation. This formulation was introduced and analyzed in [13] for the Cauchy Stokes system. In fact, this procedure, which extends analogue results for elasticity problems (see [14, 15]), has become very popular lately, especially in the context of obstacle detection [16], or coefficient reconstruction [3]. The alternating method has been applied successfully to the Cauchy Stokes problem and similar problems, see, for example $[17,18,19]$. A deep one tool relates the two approaches. Actually the Domain Decomposition type method amounts to the Euler equation of the mismuch functional. Other related methods were proposed using Steklov-Poincaré approach in [20,21] and boundary element method in [22].

This paper has two goals for the sub-Cauchy Stokes problem (1.1)-(1.5) which are analyzing the unique recovery of the lacking data on $\Gamma_{\mathrm{I}}$ from sub-Cauchy data over-specified on on $\Gamma_{\mathrm{C}}$ then solving the data completion problem. For the first goal, we have the following observation: The stream function formulation of the sub-Cauchy problem (1.1)-(1.5) is the Dirichlet problem for the biharmonic equation (see Section 2). In fact, this insight allows firstly to obtain an example where uniqueness fails to hold. On the other hand, it allows investing some recent uniqueness results in $[23,24]$ where the authors consider the uniqueness issues associated with the Dirichlet problem for the N-harmonic equations that are not covered by previously known uniqueness theorems. Based on these results we establish a local uniqueness result for the reconstruction of the pair $(\mathbf{u}, p)$, the solution of the sub-Cauchy Stokes problem (1.1)-(1.5).

For the second goal, we propose a new class of Lagrange multiplier methods to solve the Cauchy problems. The regular approaches define and compare two distinct solutions of the inverse problem, where each one 
uses one item of the given data on the accessible boundary and where the two solutions are tied together using matching conditions on the inaccessible boundary given by equality of the Dirichlet or the Neumann conditions, or both of them [13, 20] (see [15] for Robin conditions). However, more general artificial boundary conditions can be used as in domain decomposition (DD) methods (see [25, 26] for example). In the DD context, this approach assures exchanging boundary data between two problems using higher order matching conditions. Then, physically more valuable information is exchanging between the two problems and hence have better convergence behavior. Our method is based on this approach, and hence we are able to handle the data completion problem (1.1)-(1.5) in the same way. Nevertheless, in our problem $\Gamma_{\mathrm{I}}$ will play the role of the interface. As a result, using more general order 2 (or Ventcell) boundary operators, we formulate two methods each of which reduces the sub-Cauchy Stokes problem to an interfacial problem which can be solved iteratively. Both methods depend on the coupling conditions used to match the values of the unknowns on the inaccessible boundary. Furthermore, we present accelerated alternating methods for the solution of the sub-Cauchy problem based on order 2 conditions, and in which coefficients can be optimized to improve convergence rates.

The rest of this paper is organized as follows. In Section 2, we investigate the uniqueness issues for the sub-Cauchy Stokes problem. In Section 3, we present and study two Lagrange multiplier methods to solve the problem (1.1)-(1.5). The basis of the two methods is the use of the duplication process of Vogelius together with high-order domain decomposition techniques with Ventcell boundary conditions. In Section 4 of this work, we propose and analyze an optimized Dirichlet-Order 2 alternating method to solve the sub-Cauchy Stokes problem. This procedure consists in extending the alternating method to the Ventcell interfacial conditions. In the last part of this section, we invoke the Fourier computations to analyze the convergence behavior of the proposed iterative methods for one special problem in the annular domain. We compute the convergence factor for this model problem which depends on the Ventcell parameters. In Section 5, results of two-dimensional (2D) numerical experiments comparing the different methods are discussed. An application of the optimized alternating method to the fluid-dynamics of blood is given in Appendix A.

\section{Uniqueness Issues}

As explained earlier, it is clear that with the available boundary conditions the uniqueness property cannot be obtained from Fabre and Lebeau's Theorem directly. In fact, it is not clear at all what happens under the boundary conditions (1.3)-(1.4). The question to be answered is then, for homogeneous sub-Cauchy data $(\Phi, \varphi)=0$ on $\Gamma_{\mathrm{C}}$, does it follow that the only solution is the trivial one?

Some notations: For $s>1$, we designate by $H^{s}(\Omega)$ the usual Sobolev spaces. Its norm and seminorm are written $\|\cdot\|_{s, \Omega}$ and $|\cdot|_{s, \Omega}$ respectively. The space of squared integrable functions $L^{2}(\Omega)$ is endowed with a natural inner product written $(\cdot, \cdot)_{L^{2}(\Omega)}$. The associated norm is written $\|\cdot\|_{0, \Omega}$. We denote by $L_{0}^{2}(\Omega)$ the space of functions in $L^{2}(\Omega)$ with a null mean on $\Omega$. We define the Hilbert space $H_{\mathrm{D}}^{s}(\Omega)=\left\{\mathbf{v} \in H^{s}(\Omega) \mid \mathbf{v}=0\right.$ on $\left.\Gamma_{\mathrm{D}}\right\}$. For $\gamma \subset \Gamma$, we will frequently use the special space $H_{00}^{1 / 2}(\gamma)$, the set of all the restrictions to $\gamma$ of the functions of $H^{1}(\partial \Omega)$ that vanish on $\partial \Omega \backslash \gamma$. Its dual space is then denoted by $H_{00}^{-1 / 2}(\gamma)$. The associated norms are written $\|\cdot\|_{1 / 2,00, \gamma}$ and $\|\cdot\|_{-1 / 2,00, \gamma}$ respectively and $\langle\cdot, \cdot\rangle_{1 / 2,00, \gamma}$ states for the duality inner product.

\subsection{Non-uniqueness of the sub-Cauchy Stokes problem}

The main difficulty associated with the data completion problem (1.1)-(1.5) is that, no information on the pressure is available. To get rid of the pressure, we put the sub-Cauchy Stokes problem into its stream function formulation. To this aim, we use this result to calculate the shear stress component (see [27]):

Lemma 1. Let $\mathbf{u} \in H^{2}(\Omega)^{2}$ be a vector field which is tangent to $\gamma \subseteq \partial \Omega$. We then have

$$
\boldsymbol{\tau} \cdot \mathbf{D}(\mathbf{u}) \mathbf{n}=\frac{1}{2} \operatorname{curl}(\mathbf{u})+\kappa \mathbf{u} \cdot \boldsymbol{\tau} \text { on } \gamma,
$$

where $\operatorname{curl}(\mathbf{u})=\partial \mathbf{u}_{2} / \partial x_{1}-\partial \mathbf{u}_{1} / \partial x_{2}, \kappa$ is the curvature of $\gamma$ given by $d \boldsymbol{\tau} / d s=-\kappa \mathbf{n}$. 
We now define the stream function such that $\mathbf{u}=\operatorname{curl}(\psi)$, and let $\psi$ satisfy

$$
\begin{array}{lll}
\nabla^{4} \psi=0 & \text { in } \Omega, & \\
\partial_{n}^{j} \psi=0 & \text { on } \Gamma_{\mathrm{D}}, & \text { for } j=0,1, \\
\partial_{n}^{j} \psi=0 & \text { on } \Gamma_{\mathrm{C}}, & \text { for } j=0,1,2,
\end{array}
$$

where $\partial_{n}^{j}=\partial^{j} \psi / \partial^{j} \mathbf{n}$. We prove the following result

Proposition 2. Assume that $(\mathbf{u}, p) \in H^{2}(\Omega)^{2} \times H^{1}(\Omega)$ is a solution of (1.1)-(1.5) where $(\Phi, \varphi)=0$. The associated stream function of $\mathbf{u}$ is then characterized as the function $\psi \in H^{3}(\Omega)$ solution to (2.9)-(2.11).

Proof. Suppose that $(\mathbf{u}, p)$ is a solution of (1.1)-(1.5) in $H^{2}(\Omega)^{2} \times H^{1}(\Omega)$, and let $\mathbf{u}=\operatorname{curl}(\psi)$, with $\psi \in H^{3}(\Omega)$. Taking the curl of the momentum equation (1.2) is equivalent to the homogeneous biharmonic equation (2.9). Now, we turn to the boundary conditions (1.3)-(1.5). On the boundary the tangential and normal components of the velocity can be expressed as the normal and tangential derivatives of the stream function

$$
\mathbf{u} \cdot \boldsymbol{\tau}=\partial \psi / \partial \mathbf{n}, \quad \mathbf{u} \cdot \mathbf{n}=-\partial \psi / \partial \boldsymbol{\tau}
$$

The boundary conditions

$$
\psi=0 \text { ( no flow), } \quad \partial \psi / \partial \mathbf{n}=0 \text { ( no slip), }
$$

then coincide with the homogeneous Dirichlet boundary condition $\mathbf{u}=0$ on $\Gamma_{\mathrm{C}} \cup \Gamma_{\mathrm{D}}$ (see [28]). Using equation (2.8), homogeneous shear stress condition on $\Gamma_{\mathrm{C}}$ is equivalent to

$$
\Delta \psi=2 \kappa \partial \psi / \partial \mathbf{n} \quad \text { on } \Gamma_{\mathrm{C}}
$$

where $\kappa$ is the curvature of $\Gamma_{\mathrm{C}}$. Assembling the above equations, we verify the boundary conditions of the system (2.9)-(2.11), and the proof is complete.

In view of Proposition 2, it is easy to show that eliminating the pressure in the equations (1.1)-(1.5), yields a simpler equivalent formulation with only one unknown function. Another way to see this result is that the problem (2.9)-(2.11) gives a derivative representation of the boundary conditions on $\Gamma_{\mathrm{C}}$ which are more convenient than those in (1.1)-(1.5) to study Cauchy problems.

Remark 3. The unique continuation problem of (2.9)-(2.11) does not fit Holmgren's uniqueness theorem, which requires that on $\Gamma_{\mathrm{C}}$ we should have $\partial_{n}^{j} \psi=0$ on $\Gamma_{\mathrm{C}}$, for $j=0, \ldots, 3$, to ensure that $\psi$ vanishes identically. Thus, we call (2.11) a condition of vanishing sub-Cauchy data for the biharmonic equation.

Indeed, the new form of the Stokes equations with vanishing sub-Cauchy data allows us to construct explicitly an illustrative example of a non-unique solution of the original sub-Cauchy problem (1.1)-(1.5). Perhaps, unfortunately, such situation occurs, for instance, in the unit disk which is a very popular domain in numerical modeling. Let us consider

$$
\psi(z)=\frac{\left(1-|z|^{2}\right)^{3}}{|1-z|^{4}}
$$

in a unit disk domain $\mathbb{D}=\{z:|z|<1\}$ which is biharmonic and vanishes with its normal, first and second derivatives on any nontrivial arc of $\partial \mathbb{D}$ not containing the boundary singularity $z=1$ (see $[23,24]$ for more details). 


\subsection{A uniqueness result}

The above example might lead us to believe that the sub-Cauchy data (1.3)-(1.4) never gives uniqueness for the Stokes problem. In fact, this example, constructed essentially for biharmonic functions, is very special and should not be taken as generic; one can see $[23,24]$ where the authors reviewed the uniqueness theorem of Holmgren for the N-harmonic equation. As an example, the uniqueness of (2.9)-(2.11) is restored if $\Omega$ if $\Omega$ is a half-plane, or that $\Gamma_{\mathrm{C}}$ is a proper arc of a non-circular ellipse $\Omega$, then the solution to the biharmonic function $\psi$ in which all partial derivatives of $\psi$ of order $\leq 2$ vanish on $\Gamma_{\mathrm{C}}$ is unique.

Assumption 1. We suppose that $\Gamma_{\mathrm{C}}$ is a proper arc of a non-circular ellipse $\omega \subseteq \Omega$.

Now, we have this result.

Theorem 4. Assume that $(\mathbf{u}, p)$ is the solution of $(1.1)-(1.5)$ in $H^{2}(\Omega)^{2} \times H^{1}(\Omega)$ where $(\Phi, \varphi)=0$. Then, $\mathbf{u}=0$ and $p$ is constant in $\Omega$.

Proof. We have $\Gamma_{\mathrm{C}}$ is a proper arc of a non-circular ellipse $\omega \subseteq \Omega$. Then, according to Proposition 2 , the associated stream function of $\mathbf{u}$ restricted to $\omega$ is characterized by the function $\psi$ the solution of biharmonic function in which all partial derivatives of $\psi$ of order $\leq 2$ vanish on $\Gamma_{\mathrm{C}} \subset \partial \omega$. In this case, it can be readily verified that $\psi$ belongs to $H^{4}(\omega)\left[28\right.$, p. 50], and by virtue of Sobolev embedding that $\psi \in H^{4}(\omega) \subset C^{2}(\bar{\omega})$ (see [29]). Therefore the new result on the sub-Cauchy problem for the biharmonic function (see Corollary 1.8 in [23] or Corollary 8 in [24]) is applicable, and $\psi$ is unique and vanishes identically in $\omega$. Hence, $\mathbf{u}=0$ in $\omega \subseteq \Omega$. Since the work carried out by Fabre and Lebeau in [6], $\mathbf{u}=0$ and $p$ is a constant in all of $\Omega$.

Remark 5. A similar situation was addressed in [2] for an incomplete Cauchy problem. Some responses to the question of uniqueness are obtained for half-plane domains using integral representation. Note that the above result works also for half-planes (see [24]). Furthermore, domains satisfying assumption 1 are closer to the ones that we encounter in applications such as, for instance, the hamodynamics problems (see [30]).

Let us state that if the problem (1.1)-(1.5) has a unique solution this means that the sub-Cauchy data $(\Phi, \varphi)$ are compatible. Let us suppose that a weak solution $(\mathbf{u}, p)$ to problem $(1.1)-(1.5)$ exists in $H^{1}(\Omega)^{2} \times L_{0}^{2}(\Omega)$, and we assume that $(\Phi, \varphi) \in H_{00}^{1 / 2}\left(\Gamma_{\mathrm{C}}\right)^{2} \times H_{00}^{-1 / 2}\left(\Gamma_{\mathrm{C}}\right)$. Note that we can easily show that the pairs of compatible data are dense in the space $H_{00}^{1 / 2}\left(\Gamma_{\mathrm{C}}\right)^{2} \times H_{00}^{-1 / 2}\left(\Gamma_{\mathrm{C}}\right)($ see $[13,31])$.

\section{Reformulation of the sub-Cauchy Stokes problem}

In this section, we present optimized methods to solve the sub-Cauchy problem (1.1)-(1.5) with greatly enhanced convergence properties. We introduce two different well-posed problems, with a couple of solutions $\left(\mathbf{u}_{D}, p_{D}\right)$ and $\left(\mathbf{u}_{N}, p_{N}\right)$ defined in $\Omega$, both of which satisfy the Stokes equations in $\Omega$ as well as the Dirichlet condition on $\Gamma_{\mathrm{D}}$, and we attribute a Dirichlet boundary condition on $\Gamma_{\mathrm{C}}$ to the first problem while we attribute a Navier-slip condition (see Definition 6) to the second one. Basically, in order to ensure that the two solutions coincide in all of $\Omega$, Dirichlet and Neumann boundary conditions, or Robin/Fourier, should coincide on $\Gamma_{\mathrm{I}}$. For instance, in order to ensure that the two solutions coincide in all of $\Omega$, we consider matching conditions on $\Gamma_{\mathrm{I}}$ of the type

$$
-\left.\frac{\eta_{i}}{2} \Delta_{\tau} \mathbf{u}_{i}\right|_{\Gamma_{\mathrm{I}}}+\left(2 \mathbf{D}\left(\mathbf{u}_{i}\right)-p_{i}\right) \mathbf{n}+\alpha_{i} \mathbf{u}_{i}
$$

where $\Delta_{\tau}$ denotes the Laplacian operator defined on $\Gamma_{\mathrm{I}}$. The coefficients $\eta_{i}$ and $\alpha_{i}, i=D, N$, are positive constants used to specify different types of boundary conditions on $\Gamma_{\mathrm{I}}$. Either $\eta_{i}=0$, and the boundary condition is of Robin type, or we suppose $\eta_{i}>0$ and the condition will be referred to as an Order 2 or Ventcell condition. Moreover, these parameters can be chosen to optimize the convergence factor when the inverse problem is solved iteratively. This Order 2 condition involves not only the Cauchy stress and the velocity trace on $\Gamma_{\mathrm{I}}$, but also the tangential second order derivatives of the velocity on $\Gamma_{\mathrm{I}}$. This new term 
models the tangential effects of the diffusion on the inaccessible boundary (see [32] for the heat equation), and it is used here as an artificial tool to enhance the information exchange between the duplicated solutions. Specially, this order 2 operator provides clear advantages to tackle the question related to singularities of solutions at points at which geometric singularities occur, such as corners and at certain endpoints at which the boundary conditions change type. To introduce the methods we introduce the following functional space

$$
\mathcal{W}=\left\{\mathbf{u} \in H_{\mathrm{D}}^{1}(\Omega)^{2},\left.\mathbf{u}\right|_{\Gamma_{\mathrm{I}}} \in H_{0}^{1}\left(\Gamma_{\mathrm{I}}\right)^{2}\right\}
$$

where functions admit equal-order regularity both in $\Omega$ and on $\Gamma_{\mathrm{I}}$, and with a vanishing boundary value on $\Gamma_{\mathrm{D}}$. We need also to define

$$
\mathcal{W}_{\star}=\left\{\mathbf{u} \in \mathcal{W} \mid \mathbf{u}=0 \text { on } \Gamma_{\mathrm{C}}\right\} \quad \text { and } \quad \mathcal{W}_{\perp}=\left\{\mathbf{u} \in \mathcal{W} \mid \mathbf{u} \cdot \mathbf{n}=0 \text { on } \Gamma_{\mathrm{C}}\right\}
$$

Endowed with the norm

$$
\|\mathbf{u}\|_{\mathcal{W}}^{2}=\|\mathbf{u}\|_{1, \Omega}^{2}+|\mathbf{u}|_{0, \Gamma_{\mathrm{I}}}^{2}
$$

the space $\mathcal{W}$ is a Hilbert space [33]. We finally define

$$
\Lambda=L^{2}\left(\Gamma_{\mathrm{I}}\right)^{2}, \quad \Sigma=H_{00}^{1 / 2}\left(\Gamma_{\mathrm{C}}\right)^{2}, \quad \widetilde{\Sigma}=H_{00}^{-1 / 2}\left(\Gamma_{\mathrm{C}}\right) \quad \text { and } \quad M=L_{0}^{2}(\Omega) .
$$

Definition 6. A Navier-slip condition is given by the shear stress condition together with a non-penetration condition on $\Gamma_{\mathrm{C}}$, i.e., if we set $N(\mathbf{u})=(\boldsymbol{\tau} \cdot 2 \mathbf{D}(\mathbf{u}) \mathbf{n}, \mathbf{u} \cdot \mathbf{n})$ and $\boldsymbol{\varphi}=(\varphi, 0)$, then a Navier-slip condition on $\Gamma_{\mathrm{C}}$ is denoted by $N(\mathbf{u}):=\varphi$ on $\Gamma_{\mathrm{C}}$.

Remark 7. The use of a Navier slip condition in the duplication process is motivated by the fact that the shear stress condition (1.3) by itself is not a complete boundary condition for the Stokes operator. The logic behind this underspecified boundary condition is that no physical boundary condition is prescribed for the normal direction (see [2] for a similar framework).

\subsection{Method 1: Using the Ventcell-to-Dirichlet operator}

The purpose of this method is to derive and analyze a weak formulation of the sub-Cauchy Stokes problem using the Ventcell-to-Dirichlet operator. To this aim, we define the following Order 2 operator

$$
\boldsymbol{\theta}(\mathbf{u}, p)=-\left.\frac{\eta}{2} \Delta_{\tau} \mathbf{u}\right|_{\Gamma_{\mathrm{I}}}+(2 \mathbf{D}(\mathbf{u}) \mathbf{n}-p) \mathbf{n},
$$

which depends on the parameter $\eta>0$. Let $\lambda$ denotes $\left.\boldsymbol{\theta}(\mathbf{u}, p)\right|_{\Gamma_{\mathrm{I}}}$ which will be chosen as the new unknown on the inaccessible boundary $\Gamma_{\mathrm{I}}$. We now introduce a pair of functions $\left(\mathbf{u}_{D}, p_{D}\right)$ and $\left(\mathbf{u}_{N}, p_{N}\right)$ such that $\left(\mathbf{u}_{i}, p_{i}, \lambda\right) \in \mathcal{W} \times M \times \Lambda, i=D, N$, are the solutions to the following problems

$$
\begin{aligned}
\nabla \cdot \mathbf{u}_{D}=0 & \text { in } \Omega, \\
-\Delta \mathbf{u}_{D}+\nabla p_{D}=0 & \text { in } \Omega, \\
\boldsymbol{\theta}\left(\mathbf{u}_{D}, p_{D}\right)=\lambda & \text { on } \Gamma_{\mathrm{I}}, \\
\mathbf{u}_{D}=\Phi & \text { on } \Gamma_{\mathrm{C}}, \\
& \\
\nabla \cdot \mathbf{u}_{N}=0 & \text { in } \Omega, \\
-\Delta \mathbf{u}_{N}+\nabla p_{N}=0 & \text { in } \Omega, \\
\boldsymbol{\theta}\left(\mathbf{u}_{N}, p_{N}\right)=\lambda & \text { on } \Gamma_{\mathrm{I}}, \\
N\left(\mathbf{u}_{N}\right)=\boldsymbol{\varphi} & \text { on } \Gamma_{\mathrm{C}} .
\end{aligned}
$$


The key idea of this method is that we use higher order condition instead of the classical Neumann one on the inaccessible boundary $\Gamma_{I}$, leading to a coupling problem between a 2-dimensional PDE in $\Omega$ and a 1 dimensional PDE on the inaccessible boundary $\Gamma_{\mathrm{I}}$ which greatly enhance the information exchange between the duplicated solutions. The method is also motivated by the physics of the underlying problem, in which the boundary condition on the inaccessible boundary should be as "transparent" as possible to retrieve the true information. For a given $\lambda \in \Lambda$, the functions $\left(\mathbf{u}_{i}, p_{i}\right), i=D, N$, are well-posed in $\mathcal{W} \times M$, owing to the coerciveness of the associated Stokes operator together with an inf-sup condition (see subsection 3.1.1). The first variational formulation we propose is then based on the following results.

Theorem 8. Suppose that $(\mathbf{u}, p)$ is the solution of problem (1.1)-(1.5). If $p$ and $\mathbf{u}$ are sufficiently regular, then $\left(\mathbf{u}_{i}, p_{i}, \lambda\right)$, defined by

$$
\mathbf{u}_{i}=\mathbf{u}, \quad p_{i}=p, \quad \text { for } i=D, N, \quad \text { and } \lambda=-\left.\frac{\eta}{2} \Delta_{\tau} \mathbf{u}\right|_{\Gamma_{\mathrm{I}}}+(2 \mathbf{D}(\mathbf{u})-p) \mathbf{n},
$$

are respectively the unique solutions of (3.15) and (3.16) such that the following condition on the velocity traces

$$
\mathbf{u}_{N}(\lambda, \varphi)=\mathbf{u}_{D}(\lambda, \Phi), \quad \text { on } \Gamma_{\mathrm{I}}
$$

holds true.

Proof. The proof of this theorem is obvious.

Theorem 9. Assume that

$$
\left(\mathbf{u}_{i}, p_{i}, \lambda\right) \in \mathcal{W} \times M \times \Lambda, \text { for } i=D, N,
$$

are respectively the solutions of (3.15) and (3.16) such that the condition (3.18) holds true. Then, the solutions coincide in all of $\Omega$ and we have $\mathbf{u}=\mathbf{u}_{D}(\lambda, \Phi)=\mathbf{u}_{N}(\lambda, \varphi)$ and $p=p_{D}(\lambda, \Phi)=p_{N}(\lambda, \varphi)$.

Proof. Assume that (3.18) holds and let $(\mathbf{w}, q)=\left(\mathbf{u}_{D}-\mathbf{u}_{N}, p_{D}-p_{N}\right)$. Then $\mathbf{w}=0$ on $\Gamma_{\mathrm{I}}$ as well as its tangential second order derivative on $\Gamma_{\mathrm{I}}$, i.e., $-\left.\frac{\eta}{2} \Delta_{\tau} \mathbf{w}\right|_{\Gamma_{\mathrm{I}}}=0$. That $\boldsymbol{\sigma}(\mathbf{w}, q) \mathbf{n}=0$ on $\Gamma_{\mathrm{I}}$ now follows from the artificial condition on $\Gamma_{\mathrm{I}}$ (third condition of (3.15) and (3.16)). As a result, the solution $(\mathbf{w}, q)$ satisfies a Cauchy-Stokes problem with homogeneous Cauchy data on $\Gamma_{\mathrm{I}}$. Thus, we have necessarily $\mathbf{w}=0$ and $q=0$ (see $[6])$.

The above theorems are of special importance, explaining that the new unknown $\lambda \in \Lambda$ is uniquely defined on the inaccessible boundary $\Gamma_{\mathrm{I}}$ if and only if the condition (3.18) is satisfied, whence the uniqueness for problem (1.1)-(1.5) is preserved. We will show next how a variational formulation for the sub-Cauchy Stokes problem (1.1)-(1.5) can be obtained using Theorems 8 and 9 .

\subsubsection{Variational formulation}

Let $\mathscr{D}_{i}, i=D, N$, be the solution operator that associates to the boundary condition on the accessible boundary $\Gamma_{\mathrm{C}}$ and the unknown $\lambda$ on $\Gamma_{\mathrm{I}}$, the solution $\left(\mathbf{u}_{i}, p_{i}\right)$, i.e.,

$$
\begin{aligned}
& \mathscr{D}_{D}: \Lambda \times \Sigma \longrightarrow \mathcal{W} \times M \\
& (\lambda, \Phi) \mapsto\left(\mathbf{u}_{D}, p_{D}\right), \\
& \mathscr{D}_{N}: \Lambda \times \widetilde{\Sigma} \longrightarrow \mathcal{W} \times M \\
& (\lambda, \varphi) \mapsto\left(\mathbf{u}_{N}, p_{N}\right) .
\end{aligned}
$$

The variational formulation of the sub-Cauchy Stokes problem (1.1)-(1.5) uses the variational formulations of (3.15) and (3.16). With this aim, we introduce the following bilinear forms

$$
\begin{aligned}
& a_{\eta}(\mathbf{u}, \mathbf{v})=\int_{\Omega} 2 \mathbf{D}(\mathbf{u}): \mathbf{D}(\mathbf{v})+\eta \int_{\Gamma_{\mathrm{I}}} \mathbf{D}_{\tau}(\mathbf{u}): \mathbf{D}_{\tau}(\mathbf{v}), \\
& b(\mathbf{v}, q)=-\int_{\Omega} q \nabla \cdot \mathbf{v} .
\end{aligned}
$$


For a given $\lambda \in \Lambda$, the weak formulation of (3.15) reads: find $\left(\mathbf{u}_{D}, p_{D}\right) \in \mathcal{W} \times M$ to be subject to a Dirichlet condition $\left.\mathbf{u}_{D}\right|_{\Gamma_{\mathrm{C}}}=\Phi$ and satisfies

$$
\begin{aligned}
& a_{\eta}\left(\mathbf{u}_{D}, \mathbf{v}\right)+b\left(\mathbf{v}, p_{D}\right)=\int_{\Gamma_{\mathrm{I}}} \lambda \mathbf{v}, \quad \forall \mathbf{v} \in \mathcal{W}_{\star}, \\
& b\left(\mathbf{u}_{D}, q\right)=0, \quad \forall q \in M .
\end{aligned}
$$

That of problem (3.16) is as follows: find $\left(\mathbf{u}_{N}, p_{N}\right) \in \mathcal{W} \times M$ such that $\left.\mathbf{u}_{N} \cdot \mathbf{n}\right|_{\Gamma_{\mathrm{C}}}=0$ and satisfies

$$
\begin{aligned}
& a_{\eta}\left(\mathbf{u}_{N}, \mathbf{v}\right)+b\left(\mathbf{v}, p_{N}\right)=\int_{\Gamma_{\mathrm{I}}} \lambda \mathbf{v}+\langle\varphi, \mathbf{v} \cdot \boldsymbol{\tau}\rangle_{1 / 2,00, \Gamma_{\mathrm{C}}}, \quad \forall \mathbf{v} \in \mathcal{W}_{\perp}, \\
& b\left(\mathbf{u}_{N}, q\right)=0, \quad \forall q \in M .
\end{aligned}
$$

When transcribing the above variational formulations, we remark the advantage of Ventcell conditions in performing the exchange of information between the accessible and the inaccessible boundaries sharing a zero-dimensional measurement limit at their common point. Precisely, in the above formulations, we used the fact that $\mathbf{u}=0$ on $\partial \Gamma_{\mathrm{I}}$, implying that the order 2 conditions enforce that the boundary data on the common vertices of $\Gamma_{\mathrm{D}}$ and $\Gamma_{\mathrm{I}}$ to match. If, for example $\mathbf{u} \neq 0$ on $\Gamma_{\mathrm{D}}$, additional terms involving the data on $\partial \Gamma_{\mathrm{I}}$ should be added to the right-hand side of (3.19) and (3.21).

The bilinear form $a_{\eta}(\cdot, \cdot)$ is an inner product on the variational space $\mathcal{W}$ for $\eta>0$. The corresponding norm is defined by $\|\mathbf{u}\|_{\eta}=a_{\eta}(\mathbf{u}, \mathbf{u})^{1 / 2}$ for every $\mathbf{u} \in \mathcal{W}$. Precisely, we have

Lemma 10. There exist positive constants $C_{1}$, and $C_{2}$ such that

$$
C_{1}\|\mathbf{u}\|_{\mathcal{W}} \leq\|\mathbf{u}\|_{\eta} \leq C_{2}\|\mathbf{u}\|_{\mathcal{W}}, \quad \forall \mathbf{v} \in \mathcal{W}
$$

Proof. The upper bound of $a_{\eta}(\cdot, \cdot)$ is straightforward with $C_{2}=\max (2, \eta)$. The lower bound follows from Korn's inequality applied for $H_{\mathrm{D}}^{1}(\Omega)$ functions, respectively for $H_{0}^{1}\left(\Gamma_{\mathrm{I}}\right)$ functions.

The well-posedness of (3.19)-(3.20) is based on Lemma 10 and a classical inf-sup condition of Brezzi's Theorem [34], that of (3.21)-(3.22) can be ensured using the same arguments together with the analysis done in [35]. Hence the solution operators $\mathscr{D}_{i}, i=D, N$, are well-defined and in order to ensure that $\left(\mathbf{u}_{D}, p_{D}\right)=\left(\mathbf{u}_{N}, p_{N}\right)$ in all of $\Omega$, the resulting solutions are enforced to verify the equality of the velocity trace on the inaccessible boundary $\Gamma_{\mathrm{I}}$ (see Theorem 9). We introduce

$$
\left\{\begin{array} { l } 
{ ( \stackrel { \circ } { \mathbf { u } } _ { D } , \stackrel { \circ } { p } _ { D } ) = \mathscr { D } _ { D } ( 0 , \Phi ) , } \\
{ ( \stackrel { \circ } { \mathbf { u } } _ { N } , \stackrel { \circ } { p } _ { N } ) = \mathscr { D } _ { N } ( 0 , \varphi ) , }
\end{array} \quad \text { and } \quad \left\{\begin{array}{l}
\left(\overline{\mathbf{u}}_{D}, \bar{p}_{D}\right)=\mathscr{D}_{D}(\lambda, 0), \\
\left(\overline{\mathbf{u}}_{N}, \bar{p}_{N}\right)=\mathscr{D}_{N}(\lambda, 0)
\end{array}\right.\right.
$$

and the condition (3.18) between (3.15) and (3.16) is then weakly enforced through the following equation: find $\lambda \in \Lambda$ such that

$$
\int_{\Gamma_{\mathrm{I}}} \mu^{T}\left(\overline{\mathbf{u}}_{N}-\overline{\mathbf{u}}_{D}\right)(\lambda)=\int_{\Gamma_{\mathrm{I}}} \mu^{T}\left(\stackrel{\circ}{\mathbf{u}}_{D}-\stackrel{\circ}{\circ}_{N}\right), \quad \forall \mu \in \Lambda .
$$

Taking $\mathbf{v}=\overline{\mathbf{u}}_{D}(\mu)$ in (3.19)-(3.20) then $\mathbf{v}=\overline{\mathbf{u}}_{N}(\mu)$ in (3.21)-(3.22), this equation is transformed to: find $\lambda \in \Lambda$ such that

$$
s_{\eta}(\lambda, \mu)=\ell_{\eta}(\mu), \quad \forall \mu \in \Lambda,
$$

where

$$
\begin{gathered}
s_{\eta}(\lambda, \mu)=a_{\eta}\left(\overline{\mathbf{u}}_{N}(\lambda), \overline{\mathbf{u}}_{N}(\mu)\right)-a_{\eta}\left(\overline{\mathbf{u}}_{D}(\lambda), \overline{\mathbf{u}}_{D}(\mu)\right), \\
\ell_{\eta}(\mu)=a_{\eta}\left(\stackrel{\circ}{\mathbf{u}}_{D}, \overline{\mathbf{u}}_{D}(\mu)\right)-a_{\eta}\left(\stackrel{\circ}{\mathbf{u}}_{N}, \overline{\mathbf{u}}_{N}(\mu)\right)+\left\langle\varphi, \overline{\mathbf{u}}_{N}(\mu) \cdot \boldsymbol{\tau}\right\rangle_{1 / 2,00, \Gamma_{\mathrm{C}}},
\end{gathered}
$$

and both depend on the parameter $\eta$. Let $\mathcal{R}_{\eta}$ be the Ventcell-to-Dirichlet operator associated with the bilinear form $s_{\eta}(\cdot, \cdot)$. Then, we can rewrite $(3.23)$ as: find $\lambda \in \Lambda$ such that

$$
\mathcal{R}_{\eta} \lambda=\ell_{\eta}, \quad\left(\text { in } H_{0}^{1}\left(\Gamma_{\mathrm{I}}\right)^{2}\right) .
$$

After solving (3.26), we recover the solution of the sub-Cauchy Stokes problem by setting $\mathbf{u}=\overline{\mathbf{u}}_{i}(\lambda)+\stackrel{\circ}{\mathbf{u}}_{i}$ and $p=\bar{p}_{i}(\lambda)+\stackrel{\circ}{p}_{i}, i=D$ or $N$. 


\subsubsection{The analysis of the interfacial equation}

We come now to the analysis of the interfacial problem.

Lemma 11. The bilinear form $s_{\eta}(\cdot, \cdot)$ is symmetric, positive and definite, i.e.,

$$
s_{\eta}(\mu, \mu)>0, \quad \forall \mu \in \Lambda, \quad \mu \neq 0 .
$$

Proof. The proof is based on the well-posedness of (3.15) and (3.16) in $\mathcal{W} \times M$, and the uniqueness Theorem 4. Let $\mu \in \Lambda$, then the solution $\overline{\mathbf{u}}_{N}(\mu)$ can be sought as the solution of the minimization problem

$$
\overline{\mathbf{u}}_{N}(\mu)=\min _{\substack{\mathbf{w} \in \mathcal{W}_{\perp} \\ \nabla \cdot \mathbf{w}=0}} \frac{1}{2} a_{\eta}(\mathbf{w}, \mathbf{w})-\int_{\Gamma_{\mathrm{I}}} \mu \mathbf{w} .
$$

As $\overline{\mathbf{u}}_{D}(\mu) \in \mathcal{W}_{\star}$ is admissible, then

$$
\frac{1}{2}\left\|\overline{\mathbf{u}}_{N}(\mu)\right\|_{\eta}^{2}-\int_{\Gamma_{\mathrm{I}}} \mu \overline{\mathbf{u}}_{N}(\mu) \leq \frac{1}{2}\left\|\overline{\mathbf{u}}_{D}(\mu)\right\|_{\eta}^{2}-\int_{\Gamma_{\mathrm{I}}} \mu \overline{\mathbf{u}}_{D}(\mu)
$$

Using the variational formulations (3.19)-(3.20) and (3.21)-(3.22), we obtain

$$
\left\|\overline{\mathbf{u}}_{D}(\mu)\right\|_{\eta}^{2}=\int_{\Gamma_{\mathrm{I}}} \mu \overline{\mathbf{u}}_{D}(\mu), \text { and }\left\|\overline{\mathbf{u}}_{N}(\mu)\right\|_{\eta}^{2}=\int_{\Gamma_{\mathrm{I}}} \mu \overline{\mathbf{u}}_{N}(\mu)
$$

Substituting in (3.28), we obtain $s_{\eta}(\mu, \mu) \geq 0$. Now, let $s_{\eta}(\mu, \mu)=0$, from (3.24) we obtain $\left\|\overline{\mathbf{u}}_{D}(\mu)\right\|_{\eta}=$ $\left\|\overline{\mathbf{u}}_{N}(\mu)\right\|_{\eta}$. It follows from the uniqueness of the minimization problem (3.27) and Lemma 10, that $\overline{\mathbf{u}}_{D}(\mu)=$ $\overline{\mathbf{u}}_{N}(\mu)=\mathbf{v}$. Then the pair $(\mathbf{v}, q)$ with $q=\bar{p}_{D}(\mu)$ or $\bar{p}_{N}(\mu)$ is the solution of (1.1)-(1.5) with vanishing subCauchy data. As for regularity results, $\mathbf{v}$ is such that $\Delta \mathbf{v} \in L^{2}(\Omega), \mathbf{v}=0$ on $\Gamma_{\mathrm{C}} \cup \Gamma_{\mathrm{D}}$ and $\mathbf{D}(\mathbf{v}) \mathbf{n}-\Delta_{\tau} \mathbf{v} \in$ $L^{2}\left(\Gamma_{\mathrm{I}}\right)$. Such a regularity result has been proved for a similar situation in [36] for more general Ventcell boundary condition (see also [33]) and the result applies here. Hence, from Theorem 4 we have $\mathbf{v}=0$ and $q$ is a constant. The fact that $q \in M$ implies that $(\mathbf{v}, q)=0$ and then that $\mu=0$.

The ill-posedness of the interfacial problem (3.26) in the Hadamard sense is due to the compactness of $\mathcal{R}_{\eta}$

Lemma 12. The Ventcell-to-Dirichlet operator $\mathcal{R}_{\eta}$ is compact.

Proof. Using the following relations

$$
\left|\mathcal{R}_{\eta} \lambda\right|_{1, \Gamma_{\mathrm{I}}}=\left\|\overline{\mathbf{u}}_{N}(\lambda)\right\|_{\eta}-\left\|\overline{\mathbf{u}}_{D}(\lambda)\right\|_{\eta} \leq\left\|\overline{\mathbf{u}}_{N}(\lambda)\right\|_{\eta} \leq C\left\|\overline{\mathbf{u}}_{N}(\lambda)\right\|_{\mathcal{W}} \leq C\|\lambda\|_{0, \Gamma_{\mathrm{I}}}
$$

and the property of the compactness of the embedding operator of $H_{0}^{1}\left(\Gamma_{\mathrm{I}}\right)$ into $L^{2}\left(\Gamma_{\mathrm{I}}\right)$, we have the compactness of the operator $\mathcal{R}_{\eta}$ acting in $L^{2}\left(\Gamma_{\mathrm{I}}\right)^{2}$.

From the continuity and the symmetry of $s_{\eta}(\cdot, \cdot)$, we deduce that $\mathcal{R}_{\eta}$ is continuous and self-adjoint in $\Lambda$.

Lemma 13. The kernel of the operator $\mathcal{R}_{\eta}$ is the trivial space $\{0\}$, and the closure of its range is the whole space $H_{0}^{1}\left(\Gamma_{\mathrm{I}}\right)^{2}$.

Proof. From Lemma 11, we have $\operatorname{Kernel}\left(\mathcal{R}_{\eta}\right)=\{0\}$. Moreover, we have $\mathcal{R}_{\eta}$ is self-adjoint and bounded in $\Lambda$, thus $\left.\overline{\operatorname{Rang}\left(\mathcal{R}_{\eta}\right.}\right)^{\perp}=\operatorname{Kernel}\left(\mathcal{R}_{\eta}\right)$, and hence $\left.\overline{\operatorname{Rang}\left(\mathcal{R}_{\eta}\right.}\right)=H_{0}^{1}\left(\Gamma_{\mathrm{I}}\right)^{2}$.

Since the bilinear form $s_{\eta}(\cdot, \cdot)$ is non-coercive in $\Lambda$ then the space $\left(\Lambda, s_{\eta}(\cdot, \cdot)\right)$ can not be a Hilbert space with respect to the energy semi-norm arising from its inner product. This space can be completed as a Hilbert space. We denote by $\Lambda_{c}$ the completed space and let $\|\cdot\|_{\Lambda_{c}}$ be the norm induced by the extension of $s_{\eta}(\cdot, \cdot)$ to $\Lambda_{c}$. Now the space $\left(\Lambda_{c},\|\cdot\|_{\Lambda_{c}}\right)$ is a Hilbert space that is continuously and densely embedded in $\Lambda$. A detailed discussion of such a space can be found in [37]. 


\subsubsection{Regularized and optimized formulation, noisy data and error estimate}

In practice, the sub-Cauchy data $(\Phi, \varphi)$ are measurements and so they are likely to be corrupted by noise, which pollutes the compatibility behavior of the measurement. Thus, a regularizing procedure is needed for the solution of the problem (3.23) and we will make use of the Tikhonov method. For a given $\delta>0$ and $\eta>0$ let

$$
s_{\delta, \eta}\left(\lambda_{\delta}, \mu\right)=s_{\eta}\left(\lambda_{\delta}, \mu\right)+\frac{\delta}{2}\left(\lambda_{\delta}, \mu\right)_{L^{2}\left(\Gamma_{\mathrm{I}}\right)}
$$

We now consider the regularized and optimized problem of finding $\lambda_{\delta} \in \Lambda$ such that

$$
s_{\eta, \delta}\left(\lambda_{\delta}, \mu\right)=\ell_{\eta}(\mu), \quad \forall \mu \in \Lambda
$$

where in that case the parameter $\eta$ can be chosen dependently on the regularization parameter $\delta$.

Lemma 14. For $(\Phi, \varphi) \in \Sigma \times \widetilde{\Sigma}$, the regularized problem (3.29) admits a unique solution $\lambda_{\delta} \in \Lambda$ and we have the estimate

$$
\left\|\lambda_{\delta}\right\|_{0, \Gamma_{\mathrm{I}}} \leq \frac{C}{\sqrt{\delta}}\left(\|\Phi\|_{1 / 2,00, \Gamma_{\mathrm{C}}}+\|\varphi\|_{-1 / 2,00, \Gamma_{\mathrm{C}}}\right)
$$

Moreover, when $\delta$ tends to $0, \lambda_{\delta}$ converges to $\lambda$, the (unique) solution of problem (3.23) in $\Lambda_{c}$, with the following estimate

$$
\left\|\lambda-\lambda_{\delta}\right\|_{\Lambda_{c}} \leq \frac{\sqrt{\delta}}{2}\|\lambda\|_{0, \Gamma_{\mathrm{I}}}
$$

Proof. The coerciveness of $s_{\eta, \delta}(\cdot, \cdot)$ in $\Lambda$ is guaranteed for $\delta>0$. This result, together with the analysis conducted on $s_{\eta}(\cdot, \cdot)$, implies that (3.29) admits a unique solution satisfying the estimate (3.30). Now, putting $\mu=\left(\lambda_{\delta}-\lambda\right) \in \Lambda$ into the variational equations (3.23) and (3.29) and substituting the first from the second we obtain the following identity

$$
s_{\eta, \delta}\left(\lambda_{\delta}-\lambda, \lambda_{\delta}-\lambda\right)=-\frac{\delta}{4}\left(\lambda, \lambda_{\delta}-\lambda\right)_{L^{2}\left(\Gamma_{\mathrm{I}}\right)} .
$$

In compact form this can be rewritten as

$$
\left\|\lambda-\lambda_{\delta}\right\|_{\Lambda_{c}}^{2}+\frac{\delta}{4}\left\|\lambda-\lambda_{\delta}\right\|_{0, \Gamma_{\mathrm{I}}}^{2}=\frac{\delta}{4}\left(\|\lambda\|_{0, \Gamma_{\mathrm{I}}}^{2}-\left\|\lambda_{\delta}\right\|_{0, \Gamma_{\mathrm{I}}}^{2}\right)
$$

which gives the convergence of $\left(\lambda_{\delta}\right)_{\delta}$ and the estimate $(3.31)$.

Next, we suppose that noisy data are given, i.e, $\left(\Phi^{\epsilon}, \varphi^{\epsilon}\right) \in \Sigma \times \widetilde{\Sigma}$. For noisy measurements, the right-hand term $\ell_{\eta}$ in (3.29) also appears in the form of a noisy version. We have the following result:

Proposition 15. There exists a constant $C>0$ independent of the data such that

$$
\left\|\lambda_{\delta}-\lambda_{\delta}^{\epsilon}\right\|_{0, \Gamma_{\mathrm{I}}} \leq \frac{C}{\sqrt{\delta}}\left(\left\|\Phi-\Phi^{\epsilon}\right\|_{1 / 2,00, \Gamma_{\mathrm{C}}}+\left\|\varphi-\varphi^{\epsilon}\right\|_{-1 / 2,00, \Gamma_{\mathrm{C}}}\right) .
$$

Proof. Using the ellipticity of the regularized bilinear form $s_{\eta, \delta}(\cdot, \cdot)$, we have

$$
\delta\left\|\lambda_{\delta}-\lambda_{\delta}^{\epsilon}\right\|_{0, \Gamma_{\mathrm{I}}}^{2} \leq \ell_{\eta}\left(\lambda_{\delta}-\lambda_{\delta}^{\epsilon}\right)-\ell_{\eta, \epsilon}\left(\lambda_{\delta}-\lambda_{\delta}^{\epsilon}\right) .
$$

From the trace theorem together with the Cauchy-Schwartz inequality, there exists $C>0$ such that

$$
\left\|\lambda_{\delta}-\lambda_{\delta}^{\epsilon}\right\|_{0, \Gamma_{\mathrm{I}}}^{2} \leq \frac{C}{\delta}\left\|\lambda_{\delta}-\lambda_{\delta}^{\epsilon}\right\|_{0, \Gamma_{\mathrm{I}}} \times\left(\left\|\mathscr{D}_{D}\left(0, \Phi-\Phi^{\epsilon}\right)\right\|_{\mathcal{W} \times M}+\left\|\mathscr{D}_{N}\left(0, \varphi-\varphi^{\epsilon}\right)\right\|_{\mathcal{W} \times M}\right) .
$$


By the stability of the solution operators $\mathscr{D}_{i}, i=D, N$, there exists $C_{1}$, and $C_{2}$ such that

$$
\left\|\lambda_{\delta}-\lambda_{\delta}^{\epsilon}\right\|_{0, \Gamma_{\mathrm{I}}} \leq \frac{C}{\sqrt{\delta}}\left(C_{1}\left\|\Phi-\Phi^{\epsilon}\right\|_{1 / 2,00, \Gamma_{\mathrm{C}}}+C_{2}\left\|\varphi-\varphi^{\epsilon}\right\|_{-1 / 2,00, \Gamma_{\mathrm{C}}}\right)
$$

and the estimate holds by setting $C=C \max \left(C_{1}, C_{2}\right)$.

We now suppose that the noise level is known and fixed to $\epsilon>0$, that is, for a positive constant $C$ we have

$$
\left\|\Phi-\Phi^{\epsilon}\right\|_{1 / 2,00, \Gamma_{\mathrm{C}}}+\left\|\varphi-\varphi^{\epsilon}\right\|_{-1 / 2,00, \Gamma_{\mathrm{C}}} \leq C \epsilon,
$$

where the constant $C$ is chosen so that

$$
\left\|\lambda_{\delta}-\lambda_{\delta}^{\epsilon}\right\|_{0, \Gamma_{\mathrm{I}}} \leq \frac{\epsilon}{\sqrt{\delta}}
$$

Choosing the parameter $\delta=\delta(\epsilon)$ guarantees the convergence of the sequence $\left(\lambda_{\delta}^{\epsilon}\right)_{\epsilon}$ toward $\lambda$ in $\Lambda$. Such an a posteriori parameter choice can lead to a regularized solution with optimal-order of accuracy, as shown in the result given next. Furthermore, an a posteriori parameter choice for $\eta$ lead to an optimized convergence if the regularized problem is solved iteratively (see subsection 4.1).

Proposition 16. If the function $\delta(\epsilon)$ satisfies both conditions

$$
\lim _{\epsilon \rightarrow 0} \delta(\epsilon)=0, \quad \lim _{\epsilon \rightarrow 0} \frac{\epsilon}{\sqrt{\delta(\epsilon)}}=0,
$$

then the solution $\lambda_{\delta}^{\epsilon}:=\lambda^{\epsilon}$ of problem (3.29) with $\ell_{\epsilon}$ instead of $\ell$, converges to $\lambda$ in $\Lambda_{c}$ when $\epsilon$ tends to 0 . Choosing $\delta(\epsilon)=\epsilon$, we have the following estimate

$$
\left\|\lambda-\lambda^{\epsilon}\right\|_{\Lambda_{c}} \leq C \epsilon^{1 / 2}
$$

Proof. Using the estimates (3.31) and (3.32), we obtain

$$
\left\|\lambda-\lambda_{\delta}^{\epsilon}\right\|_{\Lambda_{c}} \leq\left\|\lambda-\lambda_{\delta}\right\|_{\Lambda_{c}}+\left\|\lambda_{\delta}-\lambda_{\delta}^{\epsilon}\right\|_{\Lambda_{c}} \leq \frac{\epsilon}{\sqrt{\delta(\epsilon)}}+\frac{\sqrt{\delta}}{2}\|\lambda\|_{0, \Gamma_{\mathrm{I}}} .
$$

We obtain the convergence by using Lemma 14 and the properties (3.33) of the function $\delta(\epsilon)$. The estimate (3.34) is a direct result when setting $\delta(\epsilon)=\epsilon$.

\subsection{Method 2: Using the Ventcell-to-Ventcell operator}

We introduce in this method matching conditions which are more effective for the information exchange between the duplicated solutions. Precisely, we adopt more general boundary operator

$$
\Lambda_{i}(\mathbf{u}, p)=-\left.\frac{\eta_{i}}{2} \Delta_{\tau} \mathbf{u}\right|_{\Gamma_{\mathrm{I}}}+(2 \mathbf{D}(\mathbf{u})-p) \mathbf{n}+\alpha_{i} \mathbf{u},
$$

where $\eta_{i}$ and $\alpha_{i}, i=D, N$, are positive constants such that $\alpha_{D}>\alpha_{N}$ and $\eta_{D} \geq \eta_{N}$. Contrary to Method 1 , we now introduce two competitive unknowns $\Psi_{D}$ and $\Psi_{N}$ used as interfacial variables on the inaccessible boundary $\Gamma_{\mathrm{I}}$, and such that $\left(\mathbf{u}_{i}, p_{i}, \Psi_{i}\right) \in \mathcal{W} \times M \times \Lambda, i=D, N$, solves respectively

$$
\begin{aligned}
\nabla \cdot \mathbf{u}_{D} & =0 & & \text { in } \Omega, \\
-\Delta \mathbf{u}_{D}+\nabla p_{D} & =0 & & \text { in } \Omega, \\
\Lambda_{D}\left(\mathbf{u}_{D}, p_{D}\right) & =\Psi_{D} & & \text { on } \Gamma_{\mathrm{I}}, \\
\mathbf{u}_{D} & =\Phi & & \text { on } \Gamma_{\mathrm{C}},
\end{aligned}
$$




$$
\begin{aligned}
\nabla \cdot \mathbf{u}_{N} & =0 & & \text { in } \Omega, \\
-\Delta \mathbf{u}_{N}+\nabla p_{N} & =0 & & \text { in } \Omega, \\
\Lambda_{N}\left(\mathbf{u}_{N}, p_{N}\right) & =\Psi_{N} & & \text { on } \Gamma_{\mathrm{I}}, \\
N\left(\mathbf{u}_{N}\right) & =\boldsymbol{\varphi} & & \text { on } \Gamma_{\mathrm{C}} .
\end{aligned}
$$

We refer also to this method by the 2-Lagrange multiplier (2LM) method. For a given $\Psi_{i} \in \Lambda, i=D, N$, arguments similar to those used in Method 1 ensure that the functions $\left(\mathbf{u}_{i}, p_{i}\right), i=D, N$, are well defined in $\mathcal{W} \times M$. To obtain the interfacial equation of the sub-Cauchy Stokes problem (1.1)-(1.5) with this method, we give this result

Theorem 17. Under sufficient regularity, the sub-Cauchy Stokes problem (1.1)-(1.5) has a solution if and only if there exists a pair of functions $\left(\Psi_{D}, \Psi_{N}\right) \in \Lambda \times \Lambda$ satisfying the following Order 2 or Ventcell coupling conditions

$$
\begin{aligned}
& \Psi_{D}=\Lambda_{D}\left(\mathbf{u}_{N}\left(\Psi_{N}, \varphi\right), p_{N}\left(\Psi_{N}, \varphi\right)\right), \\
& \Psi_{N}=\Lambda_{N}\left(\mathbf{u}_{D}\left(\Psi_{D}, \Phi\right), p_{D}\left(\Psi_{D}, \Phi\right)\right), \quad \text { on } \Gamma_{\mathrm{I}} .
\end{aligned}
$$

If the order 2 conditions (3.38) are satisfied then the two solutions coincide in all of $\Omega$ and we have $\mathbf{u}=$ $\mathbf{u}_{D}\left(\Psi_{D}, \Phi\right)=\mathbf{u}_{N}\left(\Psi_{N}, \varphi\right)$ and $p=p_{D}\left(\Psi_{D}, \Phi\right)=p_{N}\left(\Psi_{N}, \varphi\right)$.

Proof. Suppose that conditions (3.38) are satisfied and let $(\mathbf{w}, q)=\left(\mathbf{u}_{D}-\mathbf{u}_{N}, p_{D}-p_{N}\right)$. Subtracting the first equation of (3.38) from the second, one obtain that $\mathbf{w} \in H_{0}^{1}\left(\Gamma_{\mathrm{I}}\right)^{2}$ is the solution of

$$
-\frac{\eta_{D}-\eta_{N}}{2} \Delta_{\tau} \mathbf{w}+\left(\alpha_{D}-\alpha_{N}\right) \mathbf{w}=0, \quad \text { in } \Gamma_{\mathrm{I}} .
$$

We now seek the unique solution of this boundary problem, which is necessarily the solution $\mathbf{w}=0$ on $\Gamma_{\mathrm{I}}$ (see [38]). That $\boldsymbol{\sigma}(\mathbf{w}, q) \mathbf{n}=0$ on $\Gamma_{\mathrm{I}}$ now follows from either equation of (3.38). Thus, the difference ( $\left.\mathbf{w}, q\right)$ satisfies a Cauchy-Stokes problem with homogeneous Cauchy data on $\Gamma_{\mathrm{I}}$. A a result, $\mathbf{w}=0$ and $q=0$ in all of $\Omega$. The converse sense is obvious if a sufficiently regular solution $(\mathbf{u}, p)$ exist.

\subsubsection{Variational formulation}

In this method, we use the coupling conditions (3.38) to obtain a weak formulation for the sub-Cauchy Stokes problem (1.1)-(1.5). This is quite possible by using the two unknowns $\Psi_{D}$ and $\Psi_{N}$ used as coupling variables on the inaccessible boundary $\Gamma_{\mathrm{I}}$, and where both conditions (3.38) are written in a weak form. To this aim, we will need the following solution operators:

$$
\begin{aligned}
& \mathscr{R}_{D}: \Lambda \times \Sigma \longrightarrow \mathcal{W} \times M \\
& (\Psi, \Phi) \mapsto\left(\mathbf{u}_{D}, p_{D}\right), \\
& \mathscr{R}_{N}: \Lambda \times \widetilde{\Sigma} \longrightarrow \mathcal{W} \times M \\
& (\Psi, \varphi) \mapsto\left(\mathbf{u}_{N}, p_{N}\right) .
\end{aligned}
$$

An extension of the arguments used in Method 1 (to the case of fully Ventcell boundary conditions) guarantees that the operators $\mathscr{R}_{i}, i=D, N$, are well defined operators. Denote by $\left(\overline{\mathbf{u}}_{i}, \bar{p}_{i}\right)$ the linear part of $\mathscr{R}_{i}$ and by $\left(\stackrel{\circ}{\mathbf{u}}_{i}, \stackrel{\circ}{p}_{i}\right)$ the constant part: $\mathbf{u}_{i}=\overline{\mathbf{u}}_{i}\left(\Psi_{i}\right)+\stackrel{\circ}{\mathbf{u}}_{i}$ and $p_{i}=\bar{p}_{i}\left(\Psi_{i}\right)+\stackrel{\circ}{p}_{i}, i=D$ or $N$. We define the following Ventcell-to-Ventcell operators, which depends on the parameters $\alpha_{i}$ and $\eta_{i}, i=D, N$ :

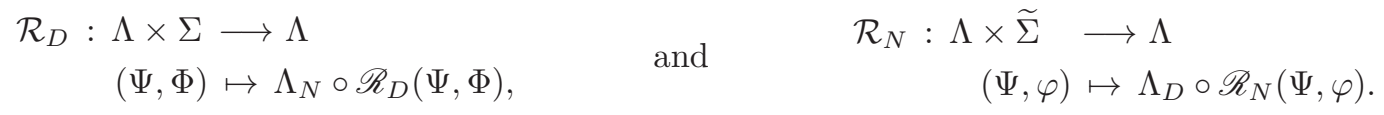

Define

$$
\begin{aligned}
& \mathcal{R}_{2 L M}: \Lambda \times \Lambda \longrightarrow \Lambda \times \Lambda \\
&\left(\begin{array}{c}
\Psi_{D} \\
\Psi_{N}
\end{array}\right) \mapsto\left(\begin{array}{c}
\Psi_{D}-\mathcal{R}_{D}\left(\Psi_{N}, 0\right) \\
\Psi_{N}-\mathcal{R}_{N}\left(\Psi_{D}, 0\right)
\end{array}\right),
\end{aligned}
$$


and

$$
\begin{aligned}
\chi: \Sigma \times \widetilde{\Sigma} & \longrightarrow \Lambda \times \Lambda \\
(\Phi, \varphi) & \mapsto\left(\begin{array}{c}
\mathcal{R}_{D}(0, \varphi) \\
\mathcal{R}_{N}(0, \Phi)
\end{array}\right) .
\end{aligned}
$$

We can write our second interfacial problem for the sub-Cauchy Stokes problem (1.1)-(1.5) as

$$
\mathcal{R}_{2 L M}\left(\begin{array}{c}
\Psi_{D} \\
\Psi_{N}
\end{array}\right)=\chi(\Phi, \varphi), \quad \text { on } \Gamma_{\mathrm{I}} .
$$

We then write (3.40) in weak form as: find $\left(\Psi_{D}, \Psi_{N}\right) \in \Lambda \times \Lambda$ such that

$$
\int_{\Gamma_{\mathrm{I}}} \mathcal{R}_{2 L M}\left(\begin{array}{c}
\Psi_{D} \\
\Psi_{N}
\end{array}\right) \cdot\left(\begin{array}{c}
\zeta_{D} \\
\zeta_{N}
\end{array}\right)=\int_{\Gamma_{\mathrm{I}}} \chi(\Phi, \varphi) \cdot\left(\begin{array}{c}
\zeta_{D} \\
\zeta_{N}
\end{array}\right), \quad \forall\left(\zeta_{D}, \zeta_{N}\right) \in \Lambda \times \Lambda .
$$

In order to study the interfacial operator $\mathcal{R}_{2 L M}$, we introduce the bilinear form associated to the Stokes problems (3.36) and (3.37):

$$
a_{\eta_{i}, \alpha_{i}}(\mathbf{u}, \mathbf{v})=\int_{\Omega} 2 \mathbf{D}(\mathbf{u}): \mathbf{D}(\mathbf{v})+\eta_{i} \int_{\Gamma_{\mathrm{I}}} \mathbf{D}_{\tau}(\mathbf{u}): \mathbf{D}_{\tau}(\mathbf{v})+\alpha_{i} \int_{\Gamma_{\mathrm{I}}} \mathbf{u v}, \quad i=D, N .
$$

This bilinear form is an inner product on the variational space $\mathcal{W}$ for $(\alpha, \eta)>0$. We can show that the corresponding norm defined by $\|\mathbf{u}\|_{\eta_{i}, \alpha_{i}}=a_{\eta_{i}, \alpha_{i}}(\mathbf{u}, \mathbf{u})^{1 / 2}$ for every $\mathbf{u} \in \mathcal{W}$ is an equivalent norm in $\mathcal{W}$. For the sake of simplicity, we consider $\eta_{D}=\eta_{N}$, then using the weak formulations of the duplicated problems (3.36) and (3.37) and with the help of the above definitions, we find out

$$
\begin{aligned}
& \int_{\Gamma_{\mathrm{I}}} \mathcal{R}_{2 L M}\left(\begin{array}{c}
\Psi_{D} \\
\Psi_{N}
\end{array}\right) \cdot\left(\begin{array}{c}
\zeta_{D} \\
\zeta_{N}
\end{array}\right)=\int_{\Gamma_{\mathrm{I}}}\left(\Psi_{D}-\Psi_{N}\right)\left(\zeta_{\mathrm{D}}-\zeta_{N}\right) \\
& -\sum_{i=\{D, N\}}\left(\alpha_{j}-\alpha_{i}\right) a_{\eta_{i}, \alpha_{i}}\left(\overline{\mathbf{u}}_{i}\left(\Psi_{i}\right), \overline{\mathbf{u}}_{i}\left(\zeta_{j}\right)\right), \quad j \neq i, \\
& \int_{\Gamma_{\mathrm{I}}} \chi(\Phi, \varphi) \cdot\left(\begin{array}{c}
\zeta_{D} \\
\zeta_{N}
\end{array}\right)=\sum_{i=\{D, N\}}\left(\alpha_{j}-\alpha_{i}\right) a_{\eta_{i}, \alpha_{i}}\left(\stackrel{\mathbf{u}}{i}_{i}, \overline{\mathbf{u}}_{i}\left(\zeta_{j}\right)\right) \\
& -\left(\alpha_{D}-\alpha_{N}\right)\left\langle\varphi, \overline{\mathbf{u}}_{N}\left(\zeta_{D}\right) \cdot \boldsymbol{\tau}\right\rangle_{1 / 2,00, \Gamma_{\mathrm{C}}}, \quad j \neq i .
\end{aligned}
$$

Contrary to Method 1, we obtain a non-symmetric interfacial operator $\mathcal{R}_{2 L M}$ but also not positive definite. GMRES or GS iterations seems well suited to the solution of such non-symmetric problems (see [39, 40, 41] for example). When an iterative method is applied to solve the interfacial equation (3.40), we will see that, formulated using the second-order conditions, the interfacial unknowns $\Psi_{D}$ and $\Psi_{N}$ do cooperate to solve the sub-Cauchy Stokes problem.

\subsubsection{The Optimized Alternating Direction Iterative Methods}

If we use for example Gauss-Seidel algorithm for solving the problem (3.40), this choice leads to an alternating direction iterative method with optimized order 2 conditions (OADI):

- Constructing $\left(\mathbf{u}_{2 k}, p_{2 k}\right)$ by solving the problem (3.36), where $\Psi_{D}=\Lambda_{D}\left(\mathbf{u}_{2 k-1}, p_{2 k-1}\right)$ on $\Gamma_{\mathrm{I}}$,

- Constructing $\left(\mathbf{u}_{2 k+1}, p_{2 k+1}\right)$ by solving the problem (3.37), where we set $\Psi_{N}=\Lambda_{N}\left(\mathbf{u}_{2 k}, p_{2 k}\right)$ on $\Gamma_{\mathrm{I}}$,

- Repeat steps 1 and 2 until a prescribed stopping criterion is satisfied.

To finish the definition of the algorithm, we provide an initial guess $\Psi_{D}^{0}$. The parameters $\alpha_{i}$ and $\eta_{i}, i=D, N$, are chosen in order to improve the convergence rate of the algorithm. In classical alternating procedures, using Dirichlet/Neumann conditions inhibit the information exchange between the duplicated solutions as well as between the accessible and inaccessible boundaries and therefore slow down the convergence of the algorithm. The above alternating method leads almost to faster convergence and improved reconstructions of the inaccessible data in few iterations. Note that this approach built with order 2 conditions can lead to many other two fields alternating methods by setting extreme values for the parameters $\alpha_{i}$ and/or $\eta_{i}$. 


\subsubsection{Regularized formulation, noisy data and stopping criteria}

We suppose that noisy data $\left(\Phi^{\epsilon}, \varphi^{\epsilon}\right) \in H_{00}^{1 / 2}\left(\Gamma_{\mathrm{C}}\right)^{2} \times H_{00}^{-1 / 2}\left(\Gamma_{\mathrm{C}}\right)$ are given. The right-hand side vector in (3.40) is then contaminated by a noise, which represents the difference $\chi^{\epsilon}-\chi$. Let us suppose that a bound of the norm of the noise is known, i.e.,

$$
\left\|\chi-\chi^{\epsilon}\right\|_{0, \Gamma_{I}} \leq \epsilon
$$

and we would like to solve the equation (3.40) with the unknown right-hand side $\chi$ by computing an approximate solution of the equation

$$
\mathcal{R}_{2 L M} \Psi=\chi^{\epsilon}, \quad \text { with } \Psi=\left(\Psi_{D}, \Psi_{N}\right)^{T} .
$$

It has been observed in [40], that the GMRES method (see [42] for the ADI method) equipped with a stopping rule based on the following discrepancy principle

$$
\left\|\mathcal{R}_{2 L M} \Psi_{k}^{\epsilon}-\chi^{\epsilon}\right\|_{0, \Gamma_{\mathrm{I}}} \leq b \epsilon,
$$

is a practical regularization method (with $b>1$ fixed), and where $k=k(\epsilon)$. Study of the behavior of such methods when $\epsilon$ approaches zero can be found in [40, 42].

\section{The Optimized Dirichlet-Order 2 Alternating Method}

As stated previously, by setting extreme values for $\alpha_{i}$ and/or $\eta_{i}$, the iterates $\Psi_{D}^{k}$ and $\Psi_{N}^{k}$ in the OADI method could cooperatively solve any combination of $\left(\mathbf{u}_{i}, \Delta_{\tau} \mathbf{u}_{i}, \boldsymbol{\sigma}\left(\mathbf{u}_{i}, p_{i}\right)\right)$ on the inaccessible boundary $\Gamma_{\mathrm{I}}$. For example, the alternating method can be reduced to solving alternatively the problem (3.36) with order 2 condition on $\Gamma_{\mathrm{I}}$ and the problem (3.37) with a Dirichlet condition on $\Gamma_{\mathrm{I}}$. This method corresponds to the case where we set $\eta_{N}=0$ and $\alpha_{N}=\infty$. This method referred from now to as the O2OA method is as follows:

- Constructing $\left(\mathbf{u}_{2 k}, p_{2 k}\right)$ by solving the problem (3.36), where $\Psi_{D}=\Lambda_{D}\left(\mathbf{u}_{2 k-1}, p_{2 k-1}\right)$ on $\Gamma_{\mathrm{I}}$,

- Constructing $\left(\mathbf{u}_{2 k+1}, p_{2 k+1}\right)$ by solving the problem (3.37), where we set $\mathbf{u}_{N}=\mathbf{u}_{2 k}$ on $\Gamma_{\mathrm{I}}$,

- Repeat steps 1 and 2 until a prescribed stopping criterion is satisfied.

In [17], an alternating method was proposed which necessarily have to exchange Dirichlet and Neumann traces at the inaccessible boundary in order to allow for convergence to the solution of the Cauchy Stokes problem. This procedure is time-consuming and leads almost to non-accurate reconstructions of the stress force. We will show next that our method produces a faster and optimized method with improved reconstructions of the inaccessible data compared to these alternating methods. At the end of this section, we will see that when the parameters are optimized, the method converges as fast as possible.

Theorem 18. Let $(\Phi, \varphi) \in \Sigma \times \widetilde{\Sigma}$ and suppose that the problem (1.1)-(1.5) has a solution in $\mathcal{W} \times M$. Let $\left(\mathbf{u}_{k}, p_{k}\right)$ be the $k$-th approximate solution in the alternating procedure described previously. Then

$$
\lim _{k \rightarrow \infty}\left\|\mathbf{u}-\mathbf{u}_{k}\right\|_{\mathcal{W}}=0 \text { and } \lim _{k \rightarrow \infty}\left\|p-p_{k}\right\|_{M}=0 .
$$

For the proof of this theorem, we will show that the O2OA method is a fixed point resolution of an interfacial problem. To see that, we consider the first two iterations of the O2OA method, and for an initial approximation $\Psi_{D} \in \Lambda$, let

$$
\left\{\begin{array} { l } 
{ ( \stackrel { \circ } { \mathbf { u } } _ { 0 } , \stackrel { \circ } { 0 } _ { 0 } ) = \mathscr { R } _ { D } ( 0 , \Phi ) , } \\
{ ( \overline { \mathbf { u } } _ { 0 } , \overline { p } _ { 0 } ) = \mathscr { R } _ { D } ( \Psi _ { D } , 0 ) , }
\end{array} \text { and } \left\{\begin{array}{l}
\left(\stackrel{\circ}{\mathbf{u}}_{1}, \circ_{1}\right)=\mathscr{R}_{N}\left(\left.\stackrel{\circ}{\mathbf{u}}_{0}\right|_{\Gamma_{I}}, \varphi\right) \\
\left(\overline{\mathbf{u}}_{1}, \bar{p}_{1}\right)=\mathscr{R}_{N}\left(\left.\overline{\mathbf{u}}_{0}\right|_{\Gamma_{I}}, 0\right)
\end{array}\right.\right.
$$

where, in abuse of notation, $\mathscr{R}_{N}$ and $\mathscr{R}_{D}$ are the solution operators defined in (3.39) and $\mathscr{R}_{N}$ maps $H_{0}^{1}\left(\Gamma_{I}\right) \times \widetilde{\Sigma}$ into $\Lambda$. We can immediately verify, using Theorem 17 , that the iterate $\left(\mathbf{u}_{1}, p_{1}\right) \in \mathcal{W} \times M$ is a solution to 
problem (1.1)-(1.5) if and only if $\Psi_{D}$ satisfies the second order condition $\Psi_{D}=-\frac{\eta_{D}}{2} \Delta_{\tau} \mathbf{u}_{1}+\boldsymbol{\sigma}\left(\mathbf{u}_{1}, p_{1}\right) \mathbf{n}+$ $\alpha_{D} \mathbf{u}_{1}=\Lambda_{D}\left(\mathbf{u}_{1}, p_{1}\right)$. This condition is equivalent to solving the interfacial problem: find $\Psi_{D} \in \Lambda$ that satisfies

$$
\Psi_{D}=A \Psi_{D}+B
$$

where $A$ and $B$ are two composite operators defined by

$$
A \Psi_{D}=\mathcal{R}_{D}\left(\overline{\mathbf{u}}_{1}, 0\right), \quad \text { and } B=\mathcal{R}_{D}\left(\stackrel{\circ}{1}_{1}, \varphi\right) .
$$

Using the same argument from [17, 19], we obtain the following result

Corollary 19. The operator $A$ is not negative, self-adjoint, non-expansive and 1 is not an eigenvalue.

Proof. Let $\left(\overline{\mathbf{u}}_{0}, \bar{p}_{0}\right)$ and $\left(\overline{\mathbf{u}}_{1}, \bar{p}_{1}\right)$ be as given above and $\left(\overline{\mathbf{u}}_{2}, \bar{p}_{2}\right)$ such that $\left(\overline{\mathbf{u}}_{2}, \bar{p}_{2}\right)=\mathscr{R}_{D}\left(\Lambda_{D}\left(\overline{\mathbf{u}}_{1}, \bar{p}_{1}\right), 0\right)$. The elements $\left(\overline{\mathbf{w}}_{0}, \bar{q}_{0}\right),\left(\overline{\mathbf{w}}_{1}, \bar{q}_{1}\right)$, and $\left(\overline{\mathbf{w}}_{2}, \bar{q}_{2}\right)$ are then constructed in the same way but with initial Ventcell condition $\xi \in \Lambda$. Let us show first that the operator $A$ is self-adjoint and non-negative. Alternating the boundary conditions, and using the symmetry of the bilinear form $a_{\eta, \alpha}$, we obtain

$$
\begin{aligned}
\left\langle A \Psi_{D}, \xi\right\rangle & =\int_{\Gamma_{I}} \Lambda_{D}\left(\overline{\mathbf{u}}_{2}, \bar{p}_{2}\right) \overline{\mathbf{w}}_{0}=\int_{\Gamma_{I}} \Lambda_{D}\left(\overline{\mathbf{u}}_{1}, \bar{p}_{1}\right) \overline{\mathbf{w}}_{1}=a_{\eta, \alpha}\left(\overline{\mathbf{w}}_{1}, \overline{\mathbf{u}}_{1}\right) \\
& =\int_{\Gamma_{I}} \Lambda_{D}\left(\overline{\mathbf{w}}_{1}, \bar{q}_{1}\right) \overline{\mathbf{u}}_{1}=\int_{\Gamma_{I}} \Lambda_{D}\left(\overline{\mathbf{w}}_{2}, \bar{q}_{2}\right) \overline{\mathbf{u}}_{0}=\left\langle\Psi_{D}, A \xi\right\rangle .
\end{aligned}
$$

Let us suppose that 1 is an eigenvalue of $A$, then there exists $\Psi_{D} \in \Lambda$ such that $\Psi_{D}=A \Psi_{D}$, which means that $\Lambda_{D}\left(\overline{\mathbf{u}}_{1}, \bar{p}_{1}\right)=\Psi_{D}=\Lambda_{D}\left(\overline{\mathbf{u}}_{0}, \bar{p}_{0}\right)$ which also gives $\left(\overline{\mathbf{u}}_{1}, \bar{p}_{1}\right)=\left(\overline{\mathbf{u}}_{0}, \bar{p}_{0}\right)$ because of the same Cauchy data on $\Gamma_{\mathrm{I}}$. This implies that $\Psi_{D}=0$. Let us finally prove that the operator $A$ is non-expansive. If $\Psi_{D} \in \Lambda$, the sequence $\left(\left\|\overline{\mathbf{u}}_{k}\right\|_{\alpha, \eta}\right)_{k}$ is decreasing; see $[17,18]$. We then obtain $\left\|A \Psi_{D}\right\|_{0, \Gamma_{I}}=\left\|\overline{\mathbf{u}}_{2}\right\|_{\alpha, \eta} \leq\left\|\overline{\mathbf{u}}_{0}\right\|_{\alpha, \eta}=\left\|\Psi_{D}\right\|_{0, \Gamma_{I}}$.

Finally, we can state that the O2OA method converges.

End of the proof of Theorem 18. It is enough to consider the case with $(\Phi, \varphi)=0$. Then we will check that $\left(\mathbf{u}_{k}, p_{k}\right)$ converges to zero in $\mathcal{W} \times M$. Let us start the iterative method with an initial Ventcell condition $\Psi_{D}=\Lambda_{D}(\mathbf{u}, p)$. Now, with induction on $k$ we will prove that $\mathbf{u}_{2 k}\left(\Psi_{D}\right)=\mathbf{u}\left(A^{k} \Psi_{D}\right)$. This assertion is true for $k=0$. Next, we assume that this is true for $k$. Introducing $A \Psi_{D}=\Lambda_{D}\left(\mathbf{u}_{2}, p_{2}\right)$, one can easily show that $\mathbf{u}_{0}\left(A^{k+1} \Psi_{D}\right)=\mathbf{u}_{2 k+2}\left(\Psi_{D}\right)$. Since the operator $A$ is self-adjoint, non-negative, non-expansive, and 1 is not an eigenvalue, this implies that $\left\|\mathbf{u}_{2 k}\right\|_{\alpha, \eta}$ tends to zero. Using the fact that $\left(\left.\left\|\mathbf{u}_{k}\right\|\right|_{\alpha, \eta}\right)_{k}$ is decreasing $\mathbf{u}_{k}$ tends to zero in $\mathcal{W}$. It remains to prove that $p_{k}$ tends to zero in $M$. By the stability of the solution operators $\mathscr{R}_{i}, i=1,2$, there exist $C_{1}$ and $C_{2}$ such that

$$
\begin{gathered}
\left\|\mathbf{u}_{2 k+1}||||_{\mathcal{W}}+\right\| p_{2 k+1} \|_{M} \leq C_{1}\left|\mathbf{u}_{2 k}\right|_{1, \Gamma_{\mathrm{I}}}, \\
\left\|\left.\mathbf{u}_{2 k+2}\left|\left\|\left.\right|_{\mathcal{W}}+\right\| p_{2 k+2}\right|\right|_{M} \leq C_{2}\right\| \Lambda_{D}\left(\mathbf{u}_{2 k+1}, p_{2 k+1}\right) \|_{0, \Gamma_{\mathrm{I}}} .
\end{gathered}
$$

This implies from the first inequality that $p_{2 k+1}$ converges to zero in $M$, and hence from the second inequality that $p_{2 k+2}$ converges to zero in $M$ and the proof is complete.

\subsection{Convergence factor formula for computing the optimized parameters}

As emphasized previously, the convergence of the alternating method can be improved using optimized Ventcell parameters. This fact can be shown by deriving the convergence factor of the alternating method. For simplicity, let us suppose that $\Omega$ is an annular domain with double radii $(1, a)$, with $a>1$. The internal circle is $\Gamma_{\mathrm{I}}$ and the external one plays the role of $\Gamma_{\mathrm{C}}$. We also suppose that the data $(\Phi, \varphi)$ vanishes since only the error equations are needed in the analysis. Let us define by $\mathbf{e}_{\theta}$, and $\mathbf{e}_{r}$ the polar unit vectors. Then, similar to the derivation of the optimized Schwarz methods, we assume that the flow is circular, that 
is, $\mathbf{u}=u(r) \mathbf{e}_{\theta}$, so that the streamlines are circular $[43,44,45,26]$. The incompressibility condition $\nabla \cdot \mathbf{u}$ is satisfied by any flow of this form, and the Stokes equations are therefore reduced to

$$
\begin{aligned}
& \frac{\partial p}{\partial r}=0, \quad 1<r<a, \\
& -\frac{1}{r} \frac{\partial p}{\partial \theta}+\left(\frac{\partial^{2} u}{\partial r^{2}}+\frac{1}{r} \frac{\partial u}{\partial r}-\frac{u}{r^{2}}\right)=0, \quad 1<r<a .
\end{aligned}
$$

Now, $u$ is a function of $r$, so from the second equation the same must be true for $\partial p / \partial \theta$, hence from the first equation, we find that $p=$ cst, otherwise $p$ would be a multivalued function of position (different at $\theta=0$ and at $\theta=2 \pi)$. Taking $p$ with a null integral on $\Omega$ leads to $p=0$. The sub-Cauchy Stokes problem (1.1)-(1.5) simplifies then to the following ill-posed Cauchy problem

$$
\begin{array}{rlrl}
\left(\frac{\partial^{2}}{\partial r^{2}}+\frac{1}{r} \frac{\partial}{\partial r}-\frac{1}{r^{2}}\right) u & =0, & 1<r<a, \\
u & =0, \quad r=a, \\
\partial_{r} u & =0, \quad r=a .
\end{array}
$$

The O2OA method now reads, for $k \geq 1$, solve

$$
\begin{aligned}
\left(\frac{\partial^{2}}{\partial r^{2}}+\frac{1}{r} \frac{\partial}{\partial r}-\frac{1}{r^{2}}\right) u_{2 k} & =0, & & 1<r<a, \\
u_{2 k} & =0, & r & =a, \\
\left(\partial_{r}+\left(\alpha-\frac{\eta}{2} \partial_{\theta \theta}\right)\right)\left[u_{2 k} \mathbf{e}_{\theta}\right] & =\Psi_{2 k-1}, & r & =1,
\end{aligned}
$$

with $\Psi_{2 k-1}=\left(\partial_{r}+\left(\alpha-\frac{\eta}{2} \partial_{\theta \theta}\right)\right)\left[u_{2 k-1} \mathbf{e}_{\theta}\right]$, and

$$
\begin{aligned}
\left(\frac{\partial^{2}}{\partial r^{2}}+\frac{1}{r} \frac{\partial}{\partial r}-\frac{1}{r^{2}}\right) u_{2 k+1} & =0, & & 1<r<a, \\
\partial_{r} u_{2 k+1} & =0, & r & =a, \\
u_{2 k+1} & =u_{2 k}, & r & =1 .
\end{aligned}
$$

We now proceed to a Fourier transformation to analyze the convergence of the above iterative method to the zero solution. From the above problems satisfied by $u_{2 k}$ and $u_{2 k+1}$, we compute both expressions

$$
u_{2 k}(r)=A_{2 k} r+\frac{B_{2 k}}{r} \quad \text { and } \quad u_{2 k+1}(r)=A_{2 k+1} r+\frac{B_{2 k+1}}{r} .
$$

Inserting the above solutions into the Fourier transformed boundary conditions on $r=a$ (i.e. the second equations of (4.48) and (4.49)), and the Fourier transformed matching conditions on $r=1$ (i.e. the third equations of (4.48) and (4.49)), we obtain by induction

$$
\begin{aligned}
u_{2 k} & =\frac{\left(1-a^{2}\right)+\left(\alpha+\frac{\eta}{2}\right)\left(1+a^{2}\right)}{\left(1+a^{2}\right)+\left(\alpha+\frac{\eta}{2}\right)\left(1-a^{2}\right)} u_{2 k-1} \\
& =\left(\frac{\left(1-a^{2}\right)+\left(\alpha+\frac{\eta}{2}\right)\left(1+a^{2}\right)}{\left(1+a^{2}\right)+\left(\alpha+\frac{\eta}{2}\right)\left(1-a^{2}\right)}\right)\left(\frac{1-a^{2}}{1+a^{2}}\right) u_{2 k-2} \\
& =\rho^{2 k} u^{0}
\end{aligned}
$$

where the convergence factor $\rho$ is given by

$$
\rho=\left(\frac{\left(1-a^{2}\right)+\left(\alpha+\frac{\eta}{2}\right)\left(1+a^{2}\right)}{\left(1+a^{2}\right)+\left(\alpha+\frac{\eta}{2}\right)\left(1-a^{2}\right)}\right)\left(\frac{1-a^{2}}{1+a^{2}}\right) .
$$


Then, the pair of parameters $(\alpha, \eta)$ should be determined by solving the min problem

$$
\min _{(\alpha, \eta)>0}|\rho(\alpha, \eta)| .
$$

Although the numerical experiments discussed at the end of the paper are done in general domains, our study of the convergence factor and parameter optimization are based on the Fourier analysis and seems therefore limited to some geometries. The results we state may be extended to a class of two-dimensional domains that can be obtained by conformal mappings from the annular domain.

\subsection{Concluding remarks}

Computing the convergence factor of the OADI method applied to the model problem (4.47) gives

$$
\rho=\left(\frac{\left(1-a^{2}\right)+\left(\alpha_{D}+\frac{\eta_{D}}{2}\right)\left(1+a^{2}\right)}{\left(1+a^{2}\right)+\left(\alpha_{D}+\frac{\eta_{D}}{2}\right)\left(1-a^{2}\right)}\right) \times\left(\frac{\left(1+a^{2}\right)+\left(\alpha_{N}+\frac{\eta_{N}}{2}\right)\left(1-a^{2}\right)}{\left(1-a^{2}\right)+\left(\alpha_{N}+\frac{\eta_{N}}{2}\right)\left(1+a^{2}\right)}\right) .
$$

We can calculate the parameters in such a way as to minimize the above convergence factor:

$$
\min _{\left(\alpha_{i}, \eta_{i}\right)>0}\left|\rho\left(\alpha_{D}, \eta_{D}, \alpha_{N}, \eta_{N}\right)\right| .
$$

The interfacial problem of Mathod 1 can be solved using Kozlov's method (see [19, 13]) and the optimized parameters can be calculated using the same techniques.

\section{Numerical Results and Discussion}

In this section, we carry out numerical experiments in 2D to investigate the performance of the methods presented in Section 3 and 4. We first test and compare the methods on a numerical example where the overspecified sub-Cauchy data $(\Phi, \varphi)$ on the accessible boundary $\Gamma_{\mathrm{C}}$ is generated from an analytic solution. Their noisy counterparts $\left(\Phi^{\epsilon}, \varphi^{\epsilon}\right)$ are produced by adding an artificial noise $\epsilon$ ranging from 0 to 0.05 . To compare the methods, the two components of the fluid velocity and the normal stress are represented on inaccessible boundary $\Gamma_{\mathrm{I}}$. With the same analytic example, the effectiveness of our methods is next showcased for a computational domain for which the uniqueness of the solution is not guaranteed. For the final numerical experiment, we illustrate the efficiency of the proposed methods for the complete Cauchy-Stokes problem where a Cauchy data are extracted from an analytic solution of the Stokes problem.

\subsection{Test 1: Non-circular ellipse domain and convergence analysis}

In this test case, $\Omega$ is a non-circular ellipse with semi-axes $a=0.25$, and $b=0.5$. To explore the efficiency of the proposed methods, we consider the reconstruction of the velocity field $\mathbf{u}$ and the stress force $\left(\boldsymbol{\sigma}_{1}, \boldsymbol{\sigma}_{2}\right)=\boldsymbol{\sigma}(\mathbf{u}, p) \mathbf{n}$ on the inaccessible boundary $\Gamma_{\mathrm{I}}$, which is defined by

$$
\Gamma_{\mathrm{I}}=\left\{(x, y):(x / a)^{2}+(y / b)^{2}=1, x>0.25\right\},
$$

from sub-Cauchy data on the rest of the boundary $\Gamma_{\mathrm{C}}$. The exact solution is given by, cf. [13],

$$
\mathbf{u}(x, y):=\frac{1}{4 \pi}\left(\log \frac{1}{\sqrt{(x-r)^{2}+y^{2}}}+\frac{(x-r)^{2}}{(x-r)^{2}+y^{2}}, \frac{(x-r) y}{(x-r)^{2}+y^{2}}\right),
$$

and

$$
p(x, y):=\frac{1}{2 \pi} \frac{(x-r)}{(x-r)^{2}+y^{2}},
$$


and involves a singular point outside the domain for $r=0.55$. We have used an iterative process based on the conjugate gradient (CG) algorithm in Method 1 (see [13]) and the OADI algorithm in Method 2 as well as the O2OA method. We stop the iterations when the following energy-like error

$$
E=\frac{1}{2} \int_{\Omega} \boldsymbol{\sigma}\left(\mathbf{u}_{1}-\mathbf{u}_{2}\right): \nabla\left(\mathbf{u}_{1}-\mathbf{u}_{2}\right)
$$

gets below $10^{-6}$. To compare the methods, Figures 1 and 2 depict the different components of the approximated velocity field $\mathbf{u}$ and stress tensor $\boldsymbol{\sigma}$ on $\Gamma_{\mathrm{I}}$, (in blue (Method 1), magenta (Method 2) and red (O2OA method)). We can see that the reconstructed fields are almost indistinguishable, in particular,
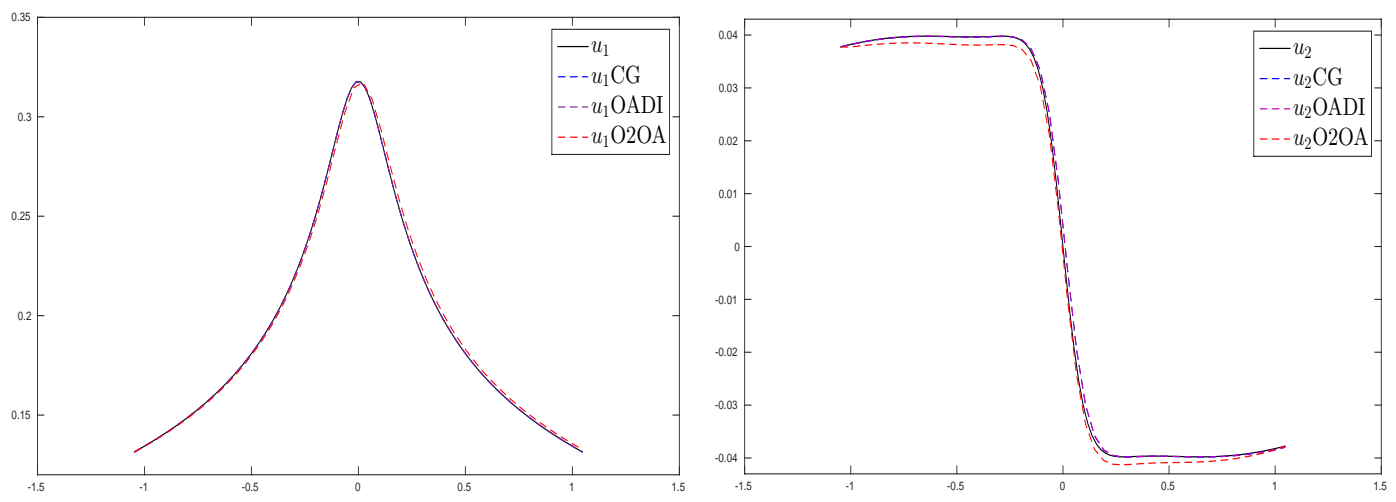

Figure 1: First test: The reconstructed velocity on $\Gamma_{\mathrm{I}}$ with $\epsilon=0$.
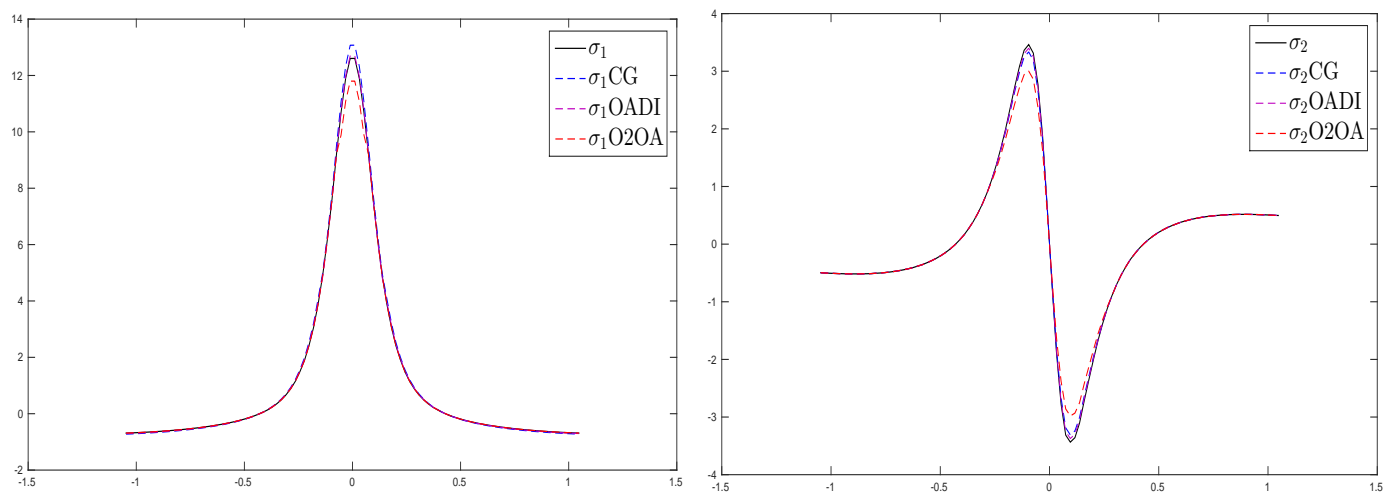

Figure 2: First test: The reconstructed stress tensor on $\Gamma_{\mathrm{I}}$ with $\epsilon=0$.

the O2OA method gives a very satisfactory result in comparison with classical alternating methods even in presence of singularities. The number of iterations of the three methods is given in Table 1, and as shown Method 1 is faster than Method 2 as well as the O2OA method. Instead of providing more figures, we only report that all the methods are faster than the classical Lagrange multiplier approaches specially in the presence of singularities. Next, we verify the performance of the O2OA method with optimized parameters. Figure 3 shows the energy-like error (in logarithmic scale) for various values of the parameters $\alpha$ and $\eta$ after 16 iterations. Clearly, we obtain a rapid convergence by taking the pair of the optimized Ventcell parameters, which are a posteriori calculated by minimizing the convergence factor (4.51). One can see that the parameters obtained are close to those giving the smallest energy error. Let us also note that the convergence can be significantly slower, if the parameters are not chosen well. 


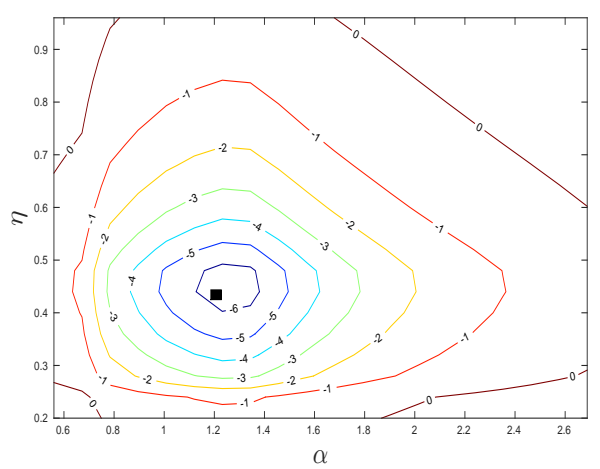

Figure 3: Level curves for the energy-like error (in logarithmic scale) after some fixed number iterations for various values of the parameters $\alpha$, and $\eta$. The square shows the optimized parameters.

\subsection{Sensitivity analysis}

We now add some noise to the data in order to test the robustness of the different methods. More precisely, only the shearing flow data $\Phi$ is polluted by a point-wise random noise with an amplitude $\epsilon=0.03$ and $\epsilon=0.05$. The shear stress data $\varphi$ remains free of noise. We use the three methods, and we stop the iterations according to the corresponding stopping criteria. We have only plotted the first component of the velocity field $\mathbf{u}_{1}$ and the first component of the stress tensor $\sigma_{1}$. We observe that the reconstruction process using the three methods remains efficient and robust for reasonable noise levels; in particular the boundary singularity is well detected. Concerning the number of iterations, the O2OA method needs more iterations than the other ones, with, though, only few iterations compared to the classical alternating method, furthermore the optimized parameters reduces the number of iterations by a significant factor.
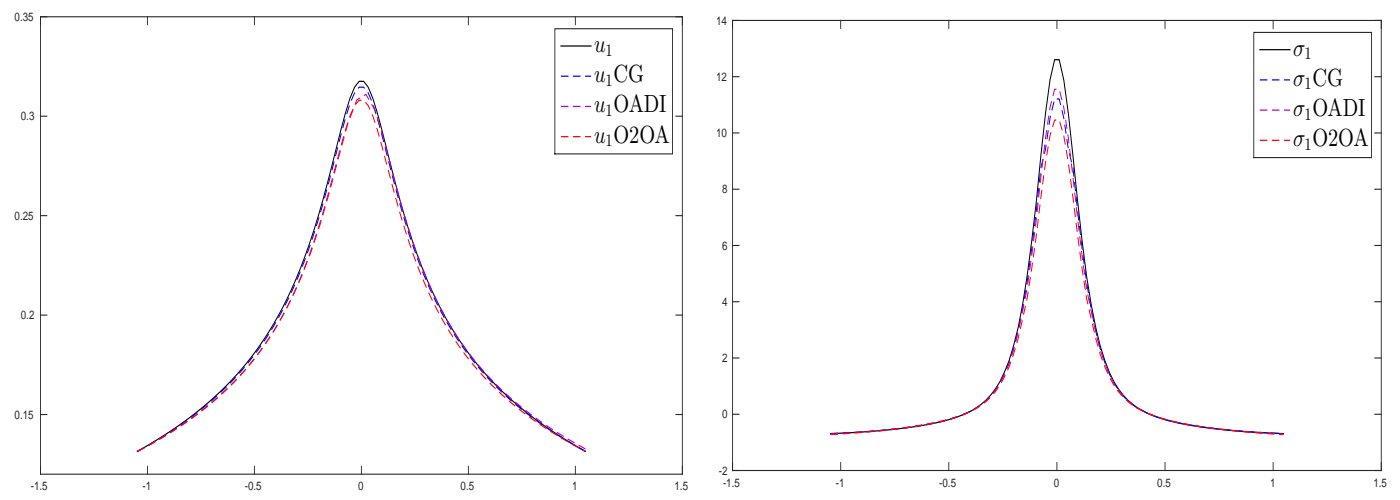

Figure 4: First test: The reconstructed first components on $\Gamma_{\mathrm{I}}$ with $\epsilon=0.03$.

\begin{tabular}{|c|ccc|}
\hline Nb of iters & Method 1 & Method 2 & O2OA method \\
$\epsilon=0$ & 14 & 14 & 16 \\
$\epsilon=0.03$ & 18 & 20 & 19 \\
$\epsilon=0.05$ & 20 & 22 & 22 \\
\hline
\end{tabular}

Table 1: The number of iterations. 

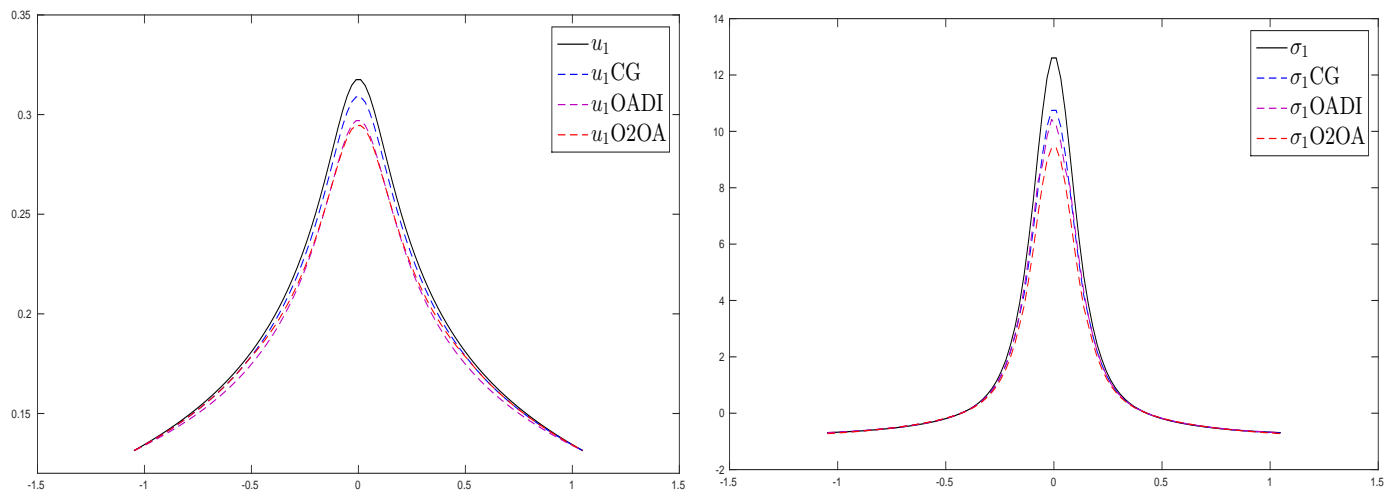

Figure 5: First test: The reconstructed first components on $\Gamma_{\mathrm{I}}$ with $\epsilon=0.05$.

\subsection{Test 2: Sub-Cauchy Stokes problem in an annular domain}

The second example focuses on the solution of problem (1.3)-(1.4) in the case of annular domains. We take the previous exact solution in $\Omega$, a two-dimensional annular domain with radii $R_{1}=2$ and $R_{2}=1$. The outer boundary is chosen to be $\Gamma_{\mathrm{C}}$ while the inner boundary is considered as $\Gamma_{\mathrm{I}}$. Let us remark that the uniqueness of the solution is not guaranteed in this case. To explore the efficiency of the proposed approaches, we consider the reconstruction of the velocity field and the stress force on the inner circle from sub-Cauchy data on the outer circle.
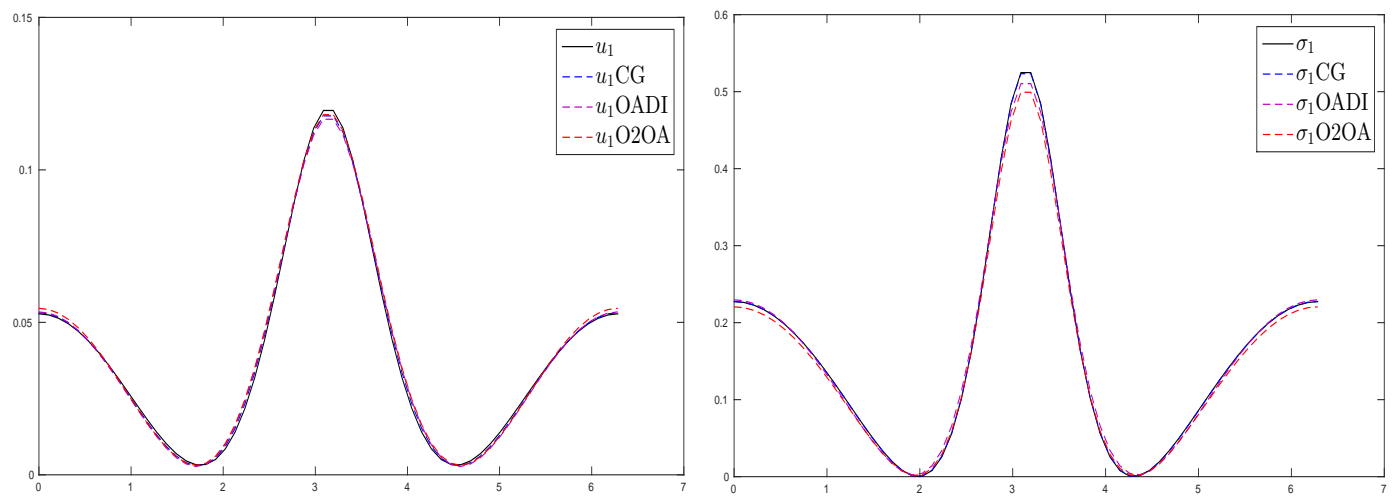

Figure 6: Second test: The reconstructed first components on the inner circle $\Gamma_{\mathrm{I}}$.

We have only plotted the first component of the velocity field $\mathbf{u}_{1}$ and the first component of the stress tensor $\boldsymbol{\sigma}_{1}$. The reconstructions obtained on the inner circle with the three procedures are given in Figure 6. They once again match quite well and the methods demonstrate the capability to select the correct solution numerically. For the O2OA method, we plot in Figure 7 the energy-like error after 20 iterations for various values of $\alpha$ and $\eta$. One can easily remark that using the optimized Ventcell parameters (marked by a square) leading to an important economy of the number of iterations. As expected, for a bad choice of the parameters the method may become significantly slower.

\subsection{Test 3: Efficiency of the methods in the case of the Cauchy Stokes problem}

In this experiment, we evaluate the effectiveness of the proposed methods in the case of the Cauchy Stokes problem. In this test case, we take a polynomial example, given by

$$
\mathbf{u}(x, y):=\left(4 y^{3}-x^{2}, 4 x^{3}+2 x y-1\right)
$$




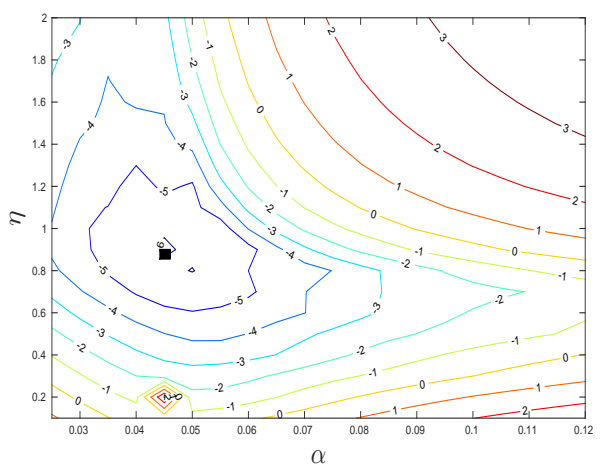

Figure 7: Level curves for the energy-like error (in logarithmic scale) after some fixed number of O2OA iterations for various values of the parameters $\alpha$, and $\eta$. The square shows the optimized parameters

and

$$
p(x, y):=24 x y-2 x,
$$

in a two-dimensional annular domain with radii $R_{1}=2$ and $R_{2}=1$. Let us precise that in that test case,
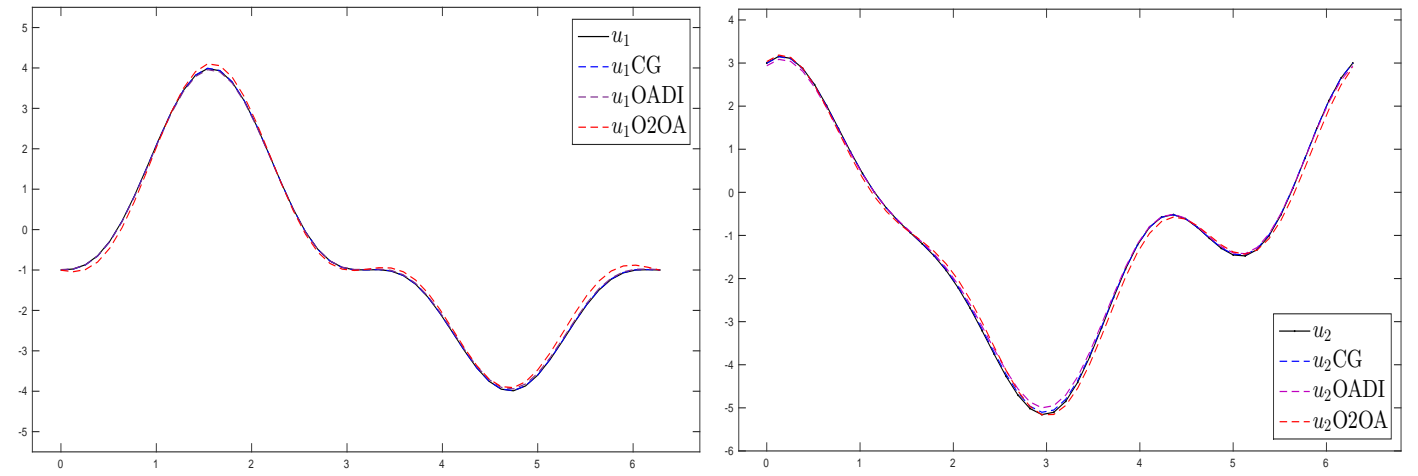

Figure 8: Third test: The reconstructed velocity on $\Gamma_{\mathrm{I}}$.
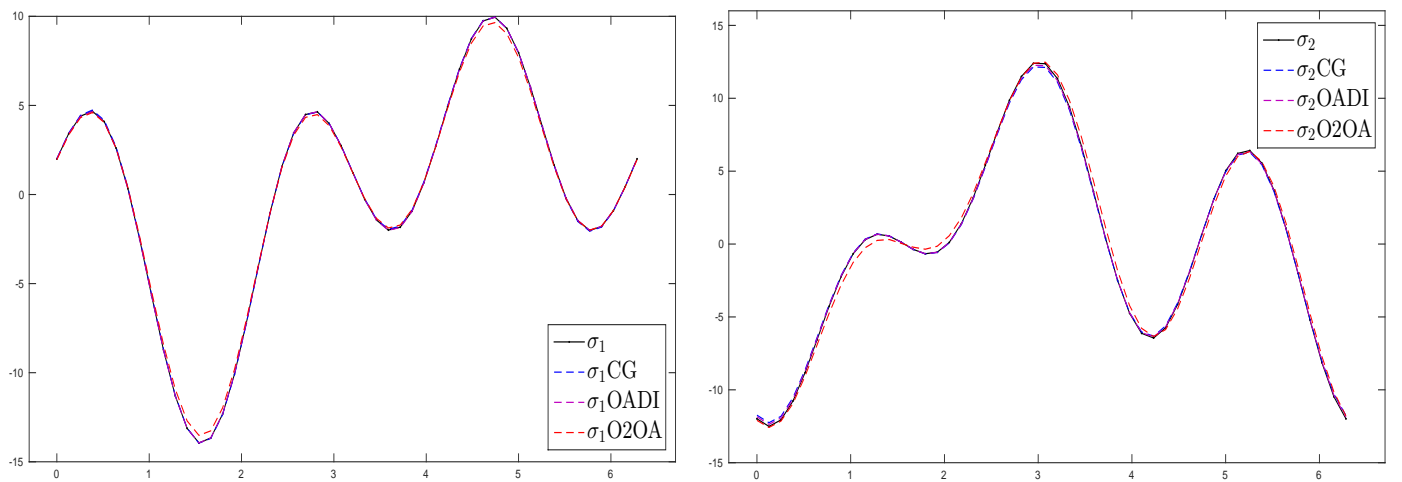

Figure 9: Third test: The reconstructed stress tensor on $\Gamma_{\mathrm{I}}$.

our task is to recover the velocity $\mathbf{u}$ and the stress force $\boldsymbol{\sigma}$ on the inner circle $\Gamma_{\mathrm{I}}$, by taking the measurements 
of Dirichlet and Neumann data on the outer circle boundary $\Gamma_{\mathrm{I}}$. The optimized parameters for the O2OA method are the same as in the previous test case. The results are visualized in Figures 8 and 9 , and it can be seen that Method 1 and Method 2 yield almost indistinguishable results; they are in close agreement with the exact solution. The results obtained using the O2OA method seem satisfactory and keep the same convergence behavior as shown in test 1 so that it needs more iterations compared to Method 1 and Method 2 (not presented).

\section{Conclusion and perspectives}

Many problems in industries have inaccessible boundary data that control the behavior of the system and need to be predicted. For this data completion problem, we have developed new methods to retrieve inaccessible data for the Stokes system from partially overdetermined boundary data. More precisely, we seek to recover missing boundary conditions on a part of the boundary for the Stokes system from the knowledge of incomplete Cauchy data given on another part of the boundary. This problem is referred to as a sub-Cauchy Stokes problem. Some answers to the question of uniqueness are obtained using the stream function representation of the Stokes equations together with the results established in [23, 24]. The missing boundary data are rephrased through interfacial equations by eliminating the interior variables. The interfacial problems are solved by iterative procedures, which requires solving well-posed Stokes problems at each iteration. The originality of the proposed approaches stems from the use of second order or Ventcell interfacial conditions, resulting in excellent reconstruction processes with considerably faster convergence rates. Numerical experiments including those with practical applications on a medical device highlight the performance of our approaches. Work underway addresses time-dependent Cauchy problems.

\section{Appendix A. A Stokes flow slipping inside an aneurysmal sac}

In this study we aim to investigate the O2OA method for the fluid-dynamics of blood in the presence of aneurysm. This anomaly is one of the most dangerous diseases of arteries, and its treatment is very difficult. In most cases the appearance and development of aneurysms proceeds for a long time without any appearing symptoms up to the moment of breaking. If the presence of an aneurysm is determined, a possible therapy consists in introducing a metallic multi-layered stent (see Figure A.10 right). This device slows down the vorticity in a aneurysm by lowering the flow velocity, pressure, its gradient, and stresses in the aneurysmal sac. An increasing effort has been made to identify hæmodynamic parameters that may play a role in the design of the optimized stent that may reduce the velocity and the oscillatory shear stress inside the sac $[46,30]$.
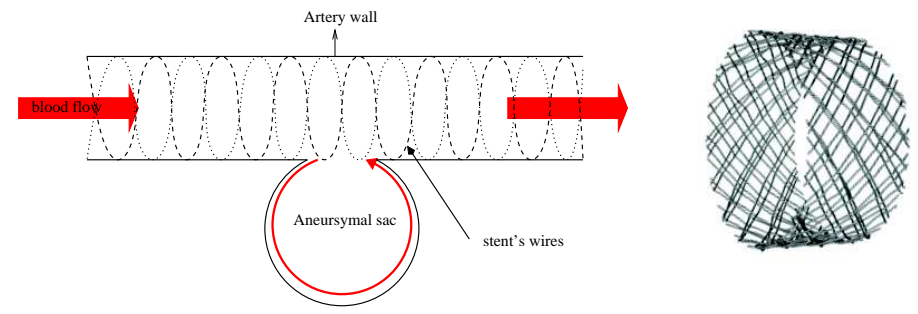

Figure A.10: A sketch of stented arteries with a aneurysmal sac (left) and a 3D example of a real metallic multi-wired stent (right).

The aim of this example is to determine the høemodynamic blood flow parameters i.e, the velocity and stress tensor, in the aperture of the aneurysmal sac in the presence of an aneurysm before and after inserting the stent (see Figure A.11). From the applicative point of view, these two situations are of interest since we identify the measurements necessary for the design of the stent, but also for post-surgical situation, where we aim to measure the efficiency of the prosthesis. In vitro, the wall shear stress value may be estimated from the flow velocity sampled near the aneurysmal wall (shearing velocity). 

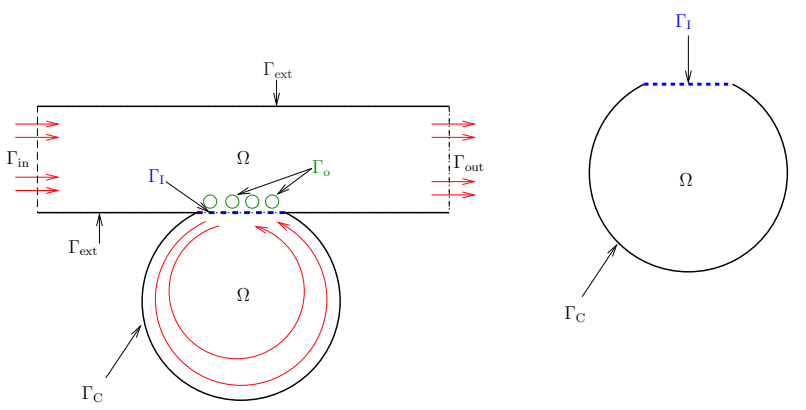

Figure A.11: A sketch of the domains for the forward problem (left) and inverse problem (right).

Then, we consider the data completion problem in the domain given by Figure A.11 (right), where the boundary is divided into two complementary parts $\Gamma_{\mathrm{C}}$ representing the aneurysmal wall and $\Gamma_{\mathrm{I}}$ representing the aperture of the sac. The sub-Cauchy data are extracted from solving the Stokes equations with the following boundary conditions

$$
\left\{\begin{array}{lll}
\boldsymbol{\sigma}(\mathbf{u}, p) \mathbf{n}=0, & \text { on } & \Gamma_{\text {out }}, \\
\mathbf{u}=\left(\frac{1}{2} y(1-y), 0\right), & \text { on } & \Gamma_{\text {in }}, \\
\mathbf{u}=0, & \text { on } & \Gamma_{\text {ext }} \cup \Gamma_{\mathrm{o}} \\
N(\mathbf{u})=(\mathbf{u} \cdot \boldsymbol{\tau}, 0), & \text { on } & \Gamma_{\mathrm{C}},
\end{array}\right.
$$

on the geometry given in Figure A.11 (left) with and without the stent ( $\Gamma_{\mathrm{o}}$ are the struts of the stent). The finite element computations of this test case are performed with FreeFem++ [47]. Figures A.12 and A.13 depict the converged solution before stenting. We observe that the method is efficient to reconstruct the inaccessible data as well to detect and locate the pressure singularities in the corners. These corners of high-pressure gradients are known to be dangerous from the medical viewpoint.
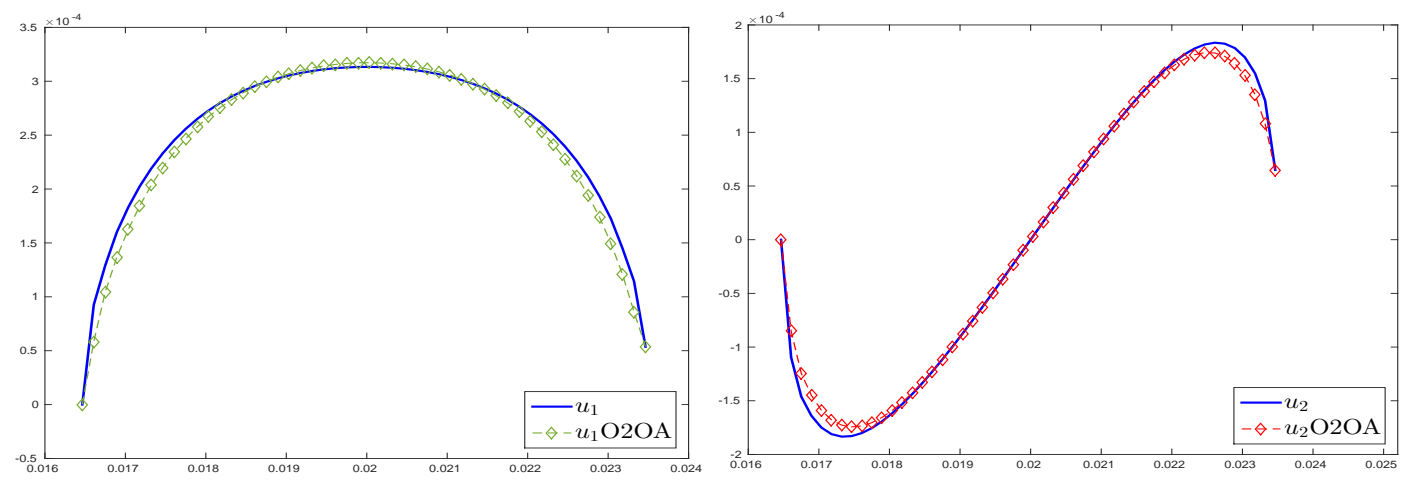

Figure A.12: Application: The reconstructed velocity on $\Gamma_{\mathrm{I}}$ (Without stent).

For the stented case, the situation is more complicated since the velocity field and the stress tensor on the inaccessible boundary are influenced by the presence of the stent. We obtain good reconstructions (see Figs.A.15 and A.16), and the method demonstrates its efficiency to detect the oscillations in the Dirichlet as well as in the Neumann boundary conditions on $\Gamma_{I}$; thanks to the Ventcell operator. These results also confirm the effect of the stent in reducing the velocity and the stress induced by the aperture of the aneurysm, but also confirm that the prosthesis promotes a more uniform distribution of the pressure and decreases the absolute value of the pressure gradient inside the sac avoiding corner singularities (see Fig.A.14 and A.17).

We can conclude that our method is robust in the sense that it solves well and consistently various complicate data completion problems. Specially the numbers of iterations remain satisfactory even in presence 

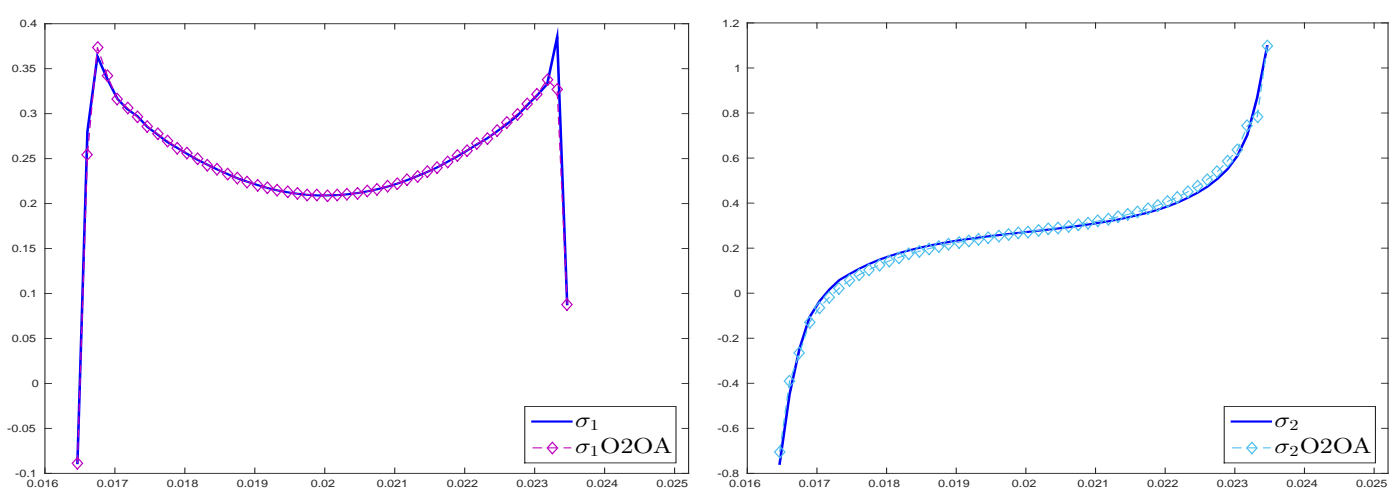

Figure A.13: Application: The reconstructed stress tensor on $\Gamma_{\mathrm{I}}$ (Without stent).
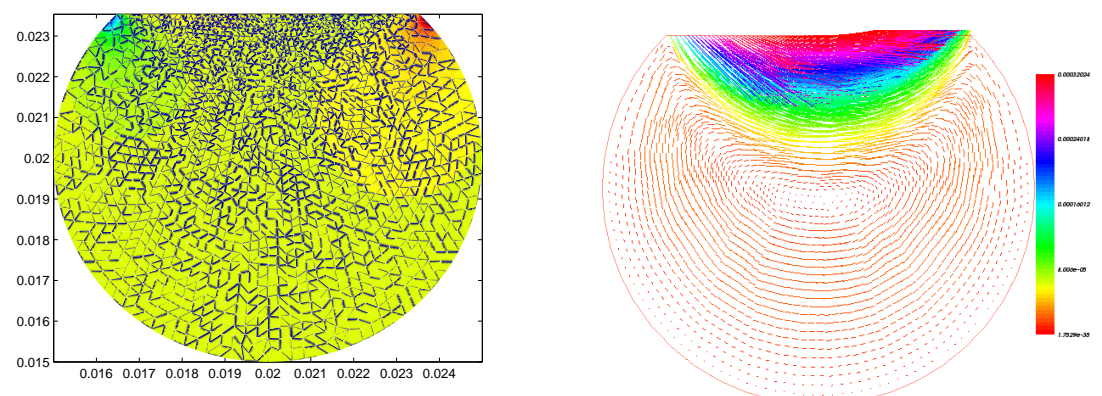

Figure A.14: Pressure and velocity in an aneurysmal sac without a stent.
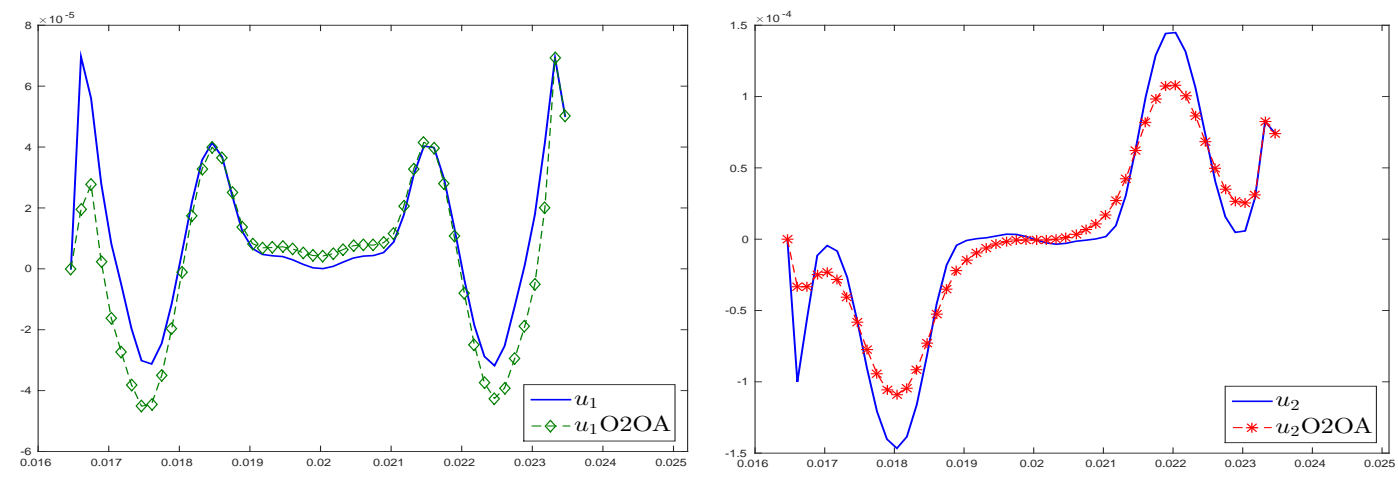

Figure A.15: Application: The reconstructed velocity on $\Gamma_{\mathrm{I}}$ (With stent).

of poor and and singular data. The convergence of the method could be improved by a good choice of the optimized parameters, with, though, this task is not known for general domains. In Figure A.18, we plot the error obtained at iteration 15 varying the parameter $\eta$. The square indicates the optimized parameter obtained using the minimization process. The square is near the small errors and leads to an optimal and faster convergence. 

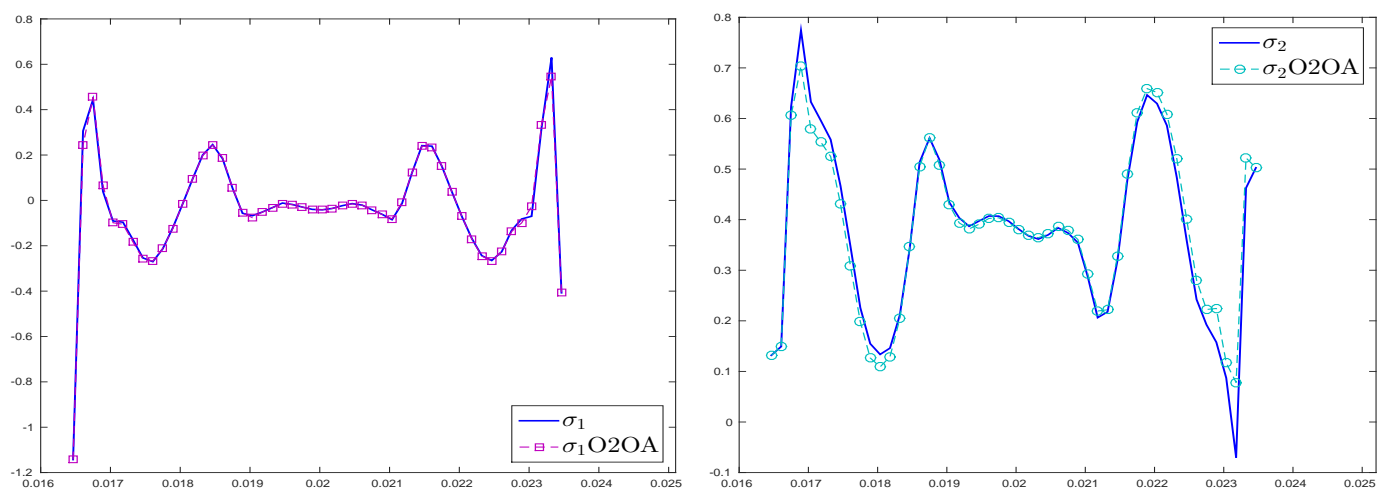

Figure A.16: Application: The reconstructed stress tensor on $\Gamma_{\mathrm{I}}$ (With stent).
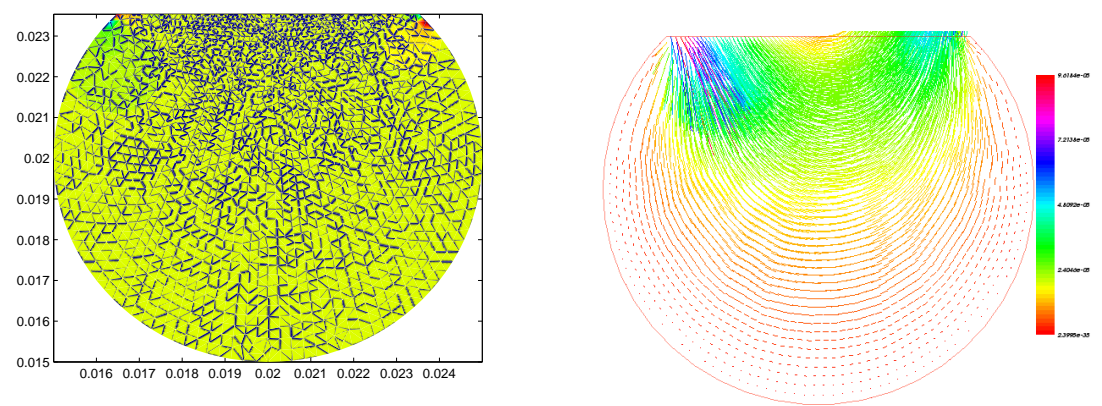

Figure A.17: Pressure and velocity in an aneurysmal sac with stent.

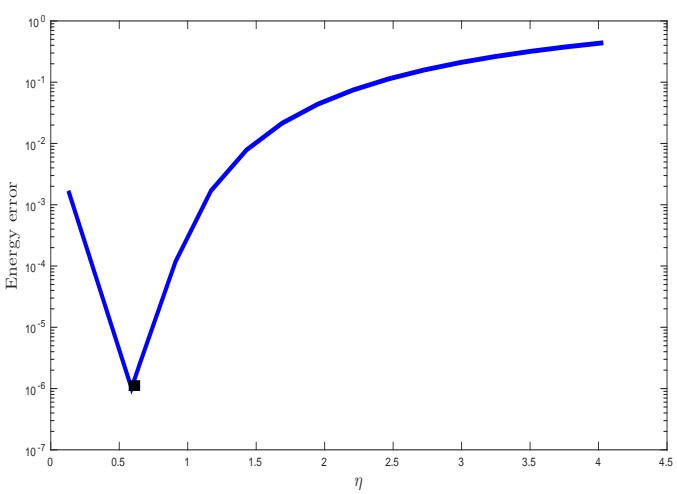

Figure A.18: Energy-like error after 15 iterations for various values of the Ventcell parameter $\eta$ ( $\alpha$ is fixed). The square shows the estimated optimized parameter.

\section{References}

[1] R. V. Kohn, M. Vogelius, Determining conductivity by boundary measurements II. Interior results, Communications on Pure and Applied Mathematics 38 (1985) 643-667.

[2] S. Andrieux, T. N. Baranger, An energy error-based method for the resolution of the Cauchy problem in 3D linear elasticity, Comput. Methods in Appl. Mech. Eng. 197 (2008) 902-920.

[3] R. J. Arthern, G. H. Gudmundsson, Initialization of ice-sheet forecasts viewed as an inverse Robin problem, J. Glaciol. 56 (2010) 527-533.

[4] Z. El Zahab, E. Divo, A. Kassab, Minimisation of the wall shear stress gradients in bypass grafts anastomoses using meshless CFD and genetic algorithms optimisation, Comput. Methods Biomech. Biomed. Eng. 13 (2010) 35-47. 
[5] Y. L. Yeow, W. C. Ko, P. P. Tang, Solving the inverse problem of Couette viscometry by Tikhonov regularization, J. Rheol. (1978-present) 44 (2000) 1335-1351.

[6] C. Fabre, Uniqueness results for stokes equations and their consequences in linear and nonlinear control problems, ESAIM Control Optim. Calc. Var. 1 (1996) 267-302.

[7] F. Caubet, Instability of an Inverse Problem for the Stationary Navier-Stokes Equations, SIAM J. Control Optim. 51 (2013) 2949-2975.

[8] C. Fabre, A. du Général de Gaulle, G. Libeau, Prolongement Unique Des Solutions, Comm. Partial Differential Equations 21 (1996) 573-596.

[9] O. Y. Imanuvilov, Remarks on exact controllability for the Navier-Stokes equations, ESAIM Control Optim. Calc. Var. 6 (2001) 39-72.

[10] T. Kim, D. Cao, Local exact controllability of the Navier-Stokes equations with the condition on the pressure on parts of the boundary, SIAM J. Control Optim. 48 (2010) 3805-3837.

[11] R. Regbaoui, Strong unique continuation for Stokes equations, Comm. Partial Differential Equations 24 (1999) $1891-1902$.

[12] R. Aboulaich, A. B. Abda, M. Kallel, A control type method for solving the Cauchy-Stokes problem, Appl. Math. Model. 37 (2013) 4295-4304.

[13] A. B. Abda, I. B. Saad, M. Hassine, Recovering boundary data: The Cauchy Stokes system, Appl. Math. Model. 37 (2013) 1-12.

[14] S. Andrieux, T. Baranger, A. B. Abda, Solving Cauchy problems by minimizing an energy-like functional, Inverse Probl. 22 (2006) 115.

[15] T. Baranger, S. Andrieux, Constitutive law gap functionals for solving the Cauchy problem for linear elliptic PDE, Appl. Math. .Comput. 218 (2011) 1970-1989.

[16] M. Badra, F. Caubet, M. Dambrine, Detecting an obstacle immersed in a fluid by shape optimization methods, Math. Mod. Meth. Appl. Sci. 21 (2011) 2069-2101.

[17] G. Bastay, T. Johansson, V. A. Kozlov, D. Lesnic, An alternating method for the stationary Stokes system, J. Appl. Math. Mech. Z. Angew. Math. Mech. 86 (2006) 268-280.

[18] F. Berntsson, V. Kozlov, L. Mpinganzima, B.-O. Turesson, An alternating iterative procedure for the Cauchy problem for the Helmholtz equation, Inverse Probl. Sci. Eng. 22 (2014) 45-62.

[19] V. A. Kozlov, V. G. Maz'ya, A. Fomin, An iterative method for solving the Cauchy problem for elliptic equations, Zhurnal Vychislitel'noi Matematiki i Matematicheskoi Fiziki 31 (1991) 64-74.

[20] F. B. Belgacem, H. E. Fekih, On Cauchy's problem: I. A variational Steklov-Poincaré theory, Inverse Probl. 21 (2005) 1915.

[21] M. L. Kadri, J. B. Abdallah, T. N. Baranger, Identification of internal cracks in a three-dimensional solid body via Steklov-Poincaré approaches, C. R. Mec. 339 (2011) 674-681.

[22] A. Zeb, L. Elliott, D. B. Ingham, D. Lesnic, Boundary element two-dimensional solution of an inverse Stokes problem, Eng. Anal. Bound. Elem. 24 (2000) 75-88.

[23] H. Hedenmalm, On the uniqueness theorem of Holmgren, Math. Z. 281 (2015) 357-378.

[24] A. Borichev, H. Hedenmalm, Weighted integrability of polyharmonic functions, Adv. Math. 264 (2014) $464-505$.

[25] P. Chevalier, F. Nataf, Symmetrized method with optimized second-order conditions for the Helmholtz equation, Contemp. Math. 218 (1998) 400-407.

[26] T. T. P. Hoang, C. Japhet, M. Kern, J. E. Roberts, Ventcell conditions with mixed formulations for flow in porous media, in: 22th International conference on domain decomposition methods, 2013.

[27] T. Clopeau, A. Mikelic, R. Robert, On the vanishing viscosity limit for the 2D incompressible Navier-Stokes equations with the friction type boundary conditions, Nonlinearity 11 (1998) 1625.

[28] V. Girault, P.-A. Raviart, Finite element methods for Navier-Stokes equations: theory and algorithms, volume 5, Springer Science \& Business Media, 2012.

[29] R. A. Adams, J. J. Fournier, Sobolev spaces, volume 140, Academic press, 2003.

[30] V. Milisic, Blood-flow modelling along and trough a braided multi-layer metallic stent, arXiv preprint arXiv:0908.2513 (2009).

[31] A. V. Fursikov, Optimal control of distributed systems. Theory and applications, American Mathematical Soc., 2000.

[32] G. R. Goldstein, et al., Derivation and physical interpretation of general boundary conditions, Adv. Differential Equations 11 (2006) 457-480.

[33] M. Dambrine, D. Kateb, Persistency of wellposedness of Ventcel's boundary value problem under shape deformations, J. Math. Anal. Appl. 394 (2012) 129-138.

[34] F. Brezzi, J. Douglas Jr, Stabilized mixed methods for the Stokes problem, Numer. Math. 53 (1988) $225-235$.

[35] D. Peterseim, S. A. Sauter, Finite element methods for the Stokes problem on complicated domains, Comput. Methods in Appl. Mech. Eng. 200 (2011) 2611-2623.

[36] J. Szeftel, Calcul pseudodifférentiel et paradifférentiel pour l'étude de conditions aux limites absorbantes et de propriétés qualitatives d'équations aux dérivées partielles non linéaires, Ph.D. thesis, 2004. URL: http://www.theses.fr/2004PA132001, thèse de doctorat dirigée par Halpern, Laurence Mathématiques appliquées Paris 132004

[37] M. Azaïez, F. B. Belgacem, H. E. Fekih, On Cauchy's problem: II. Completion, regularization and approximation, Inverse Probl. 22 (2006) 1307.

[38] F. Brezzi, M. Fortin, Mixed and Hybrid Finite Element Methods, Springer-Verlag New York, Inc., New York, NY, USA, 1991.

[39] D. Calvetti, B. Lewis, L. Reichel, On the choice of subspace for iterative methods for linear discrete ill-posed problems, 
Int. J. Appl. Math. Comput. Sci. 11 (2001) 1069-1092.

[40] D. Calvetti, B. Lewis, L. Reichel, On the regularizing properties of the GMRES method, Numer. Math. 91 (2002) 605-625.

[41] A. Neuman, L. Reichel, H. Sadok, Algorithms for range restricted iterative methods for linear discrete ill-posed problems, Numer. Algoritm. 59 (2012) 325-331.

[42] P. C. Hansen, Regularization tools: a Matlab package for analysis and solution of discrete ill-posed problems, Numer. Algoritm. 6 (1994) 1-35.

[43] M. J. Gander, Y. Xu, Optimized Schwarz methods for circular domain decompositions with overlap, SIAM J. Numer. Anal. 52 (2014) 1981-2004.

[44] M. J. Gander, Optimized schwarz methods, SIAM J. Numer. Anal. 44 (2006) 699-731.

[45] O. Dubois, Optimized Schwarz methods with Robin conditions for the advection-diffusion equation, in: Domain Decomposition Methods in Science and Engineering XVI, Springer, 2007, pp. 181-188.

[46] M. A. Fernández, J.-F. Gerbeau, V. Martin, Numerical simulation of blood flows through a porous interface, ESAIM Math. Model. Num. 42 (2008) 961-990.

[47] O. Pironneau, F. Hecht, A. Hyaric, K. Ohtsuka, FreeFEM, URL: http://www. freefem. org (2006). 